\title{
Laserwire at the Accelerator Test Facility 2 with submicrometer resolution
}

\author{
L. J. Nevay, ${ }^{*}$ S. T. Boogert, P. Karataev, and K. Kruchinin \\ John Adams Institute at Royal Holloway, University of London, Egham, TW20 OEX, United Kingdom \\ L. Corner, D. F. Howell, and R. Walczak \\ John Adams Institute at University of Oxford, Denys Wilkinson Building, \\ Oxford OX1 3RH, United Kingdom
}

A. Aryshev, J. Urakawa, and N. Terunuma

KEK, 1-1 Oho, Tsukuba, Ibaraki 305-0801, Japan

(Received 2 April 2014; published 9 July 2014)

\begin{abstract}
A laserwire transverse electron beam size measurement system has been developed and operated at the Accelerator Test Facility 2 at the High Energy Accelerator Research Organization, Japan (KEK). Special electron beam optics were developed to create an approximately $1 \times 100 \mu \mathrm{m}$ (vertical $\times$ horizontal) electron beam at the laserwire location, which was profiled using $150 \mathrm{~mJ}, 71 \mathrm{ps}$ laser pulses with a wavelength of $532 \mathrm{~nm}$. The precise characterization of the laser propagation allows the non-Gaussian laserwire scan profiles caused by the laser divergence to be deconvolved. A minimum vertical electron beam size of $1.07 \pm 0.06$ (stat) \pm 0.05 (sys) $\mu$ m was measured. A vertically focusing quadrupole just before the laserwire was varied while making laserwire measurements and the projected vertical emittance was measured to be $82.56 \pm 3.04 \mathrm{pm}$ rad.
\end{abstract}

DOI: 10.1103/PhysRevSTAB.17.072802

PACS numbers: 41.85.Ew, 29.20.Ej

\section{INTRODUCTION}

For future linear electron-positron colliders such as the Compact Linear Collider (CLIC) [1] and the International Linear Collider (ILC) [2], measurement of the particle beam emittance is essential for achieving and maintaining the required nanometer level final focus beam sizes if their target luminosity is to be reached. The emittance is typically measured by measuring the transverse profile of the beam at several points in the lattice with a different betatron phase advance, or by having a single beam size measurement location and changing the strength of an upstream quadrupole. Two commonly used methods at electron accelerators to measure the transverse beam profile are optical transition radiation (OTR) screens that image the beam directly [3] and wire scanners [4]. However, both of these devices are destructive and themselves suffer damage from high charge density beams and therefore are not suitable for making measurements at the full beam energy or bunch charge as would be required for continuous monitoring and tuning of the accelerator optics in future linear colliders [5].

A laserwire is a beam profile monitor based on Compton scattering of laser photons from the electrons or positrons

\footnotetext{
*laurie.nevay@rhul.ac.uk

Published by the American Physical Society under the terms of the Creative Commons Attribution 3.0 License. Further distribution of this work must maintain attribution to the author $(s)$ and the published article's title, journal citation, and DOI.
}

in the particle beam [6]. With high energy particle beams, the scattered photons have a high energy and travel nearly parallel to the particle beam and can be detected after a bend in the beam line, that deflects the charged particle beam. As the laser focus is scanned transversely across the particle beam, the rate of Compton-scattered photons is modulated yielding a laserwire scan. With knowledge of the laser size at its focus, the laserwire scan can be deconvolved to give the electron beam profile. As the Compton cross section is very small, a high power pulsed laser source must be used and only a small fraction of the bunch particles are scattered.

A number of laserwire beam profile monitors have been demonstrated such as those at Stanford Linear Collider (SLC) $[7,8]$ and at PETRAII in DESY [9]. In the case of the laserwire at SLC, an ultraviolet wavelength laser was used to achieve micrometer size laserwire scans. A focusing geometry consisting of both transmissive and reflective optics was chosen to compensate for spherical aberrations, allowing the micrometer size focused laser spot sizes to be achieved. However, the reflective geometry prevents measurement of the focused laser spot directly and therefore calibration as measuring the focused spot will intercept the incoming laser beam. Additionally, the laser pulse energy cannot be measured directly and strict limits are placed on the scanning range. Alternatively, transmissive focusing optics allow direct access to the laser focus for measurement and therefore laserwire calibration. It also affords a greater scanning range and allows the laser pulse energy to be measured after the interaction point for normalization 
purposes. As the minimum focused spot size of the laser beam (and therefore the resolution of the laserwire) is limited by the wavelength of light, a wavelength less than $1 \mu \mathrm{m}$ must be used to measure a $1 \mu \mathrm{m}$ electron beam. Wavelengths below $300 \mathrm{~nm}$ limit the choice of optical materials due to absorption and also necessitate a higher power laser system for the same delivered peak laser power. A good compromise is the use of a visible wavelength laser with transmissive optics [10].

The Accelerator Test Facility (ATF) is a prototype damping ring [11] with an extraction and dump line where our first laserwire system was installed [10], which measured a minimum electron beam size of $4.8 \pm 0.3 \mu \mathrm{m}$. However, it was observed that Rayleigh range effects were present and the model used to describe this requires knowledge of the horizontal electron beam size. Without this knowledge, the model leaves some ambiguity about the size of the electron beam. The extraction line was significantly upgraded to create a prototype final focus system for future linear colliders, called ATF2 [12,13]. This paper presents results from the upgraded ATF laserwire system that aims to achieve micrometer sized transverse profiles using a visible wavelength laser system. Precise laser characterization and horizontal laserwire scans allow the more detailed model to be used and accurately measure both the horizontal and veritcal electron beam sizes.

\section{SETUP}

To demonstrate the desired micrometer size profiles, the laserwire system was positioned in the matching section of the ATF2 extraction line at a virtual image point of the final focus in the vertical dimension as shown in Fig. 1. A photograph of the experimental setup in the ATF2 beam line is shown in Fig. 2. An overview of the various subsystems and their upgrades from the laserwire installation at the ATF are described in the following sections.

\section{A. ATF2 electron beam optics}

A summary of the electron beam parameters is given in Table I. During laserwire operations, a specially developed set of electron beam optics was used to minimize the vertical beam size at the laserwire interaction point (LWIP).
In Fig. 3, both sets of electron beam optics are shown for the extraction line, matching section, and beginning of the final focus. With the normal ATF2 settings, a vertical waist exists $\sim 20 \mathrm{~cm}$ downstream from the LWIP at the location of the MFB2FF cavity BPM (CBPM). The laserwire electron beam optics were designed to move the waist to the LWIP and reduce the vertical electron beam size further. Additionally, with the normal ATF2 optics, the horizontal amplitude function $\beta_{x}$ expands in the final focus section to $\beta_{x}>40 \mathrm{~km}$ as can be seen in the top part of Fig. 3, which intentionally collimates the electron beam using the beam pipe. This, however, produces a large background for the laserwire detector, and so an effort was made to significantly reduce $\beta_{x}$ in the final focus section. As expected, reducing $\beta_{x}$ increases $\beta_{y}$, but this does not generate comparable background levels as the vertical emittance is much lower than the horizontal emittance. Fig. 4 shows $\beta_{x}$ and $\beta_{y}$ around the laserwire location, where the shifted vertical waist is clear.

Using the laserwire electron beam optics, $\beta_{x}^{*}=8.822 \mathrm{~m}$ and $\beta_{y}^{*}=15.625 \mathrm{~mm}$ at the LWIP $(*$ denotes the value of functions at the LWIP), which combined with the nominal emittance values, gives a predicted electron beam size of $0.495 \mu \mathrm{m} \times 117 \mu \mathrm{m}$ (vertical $\times$ horizontal). Apart from the amplitude functions $\beta_{x, y}$, the electron beam size $\sigma_{e}$ also depends on the dispersion at the LWIP as described by

$$
\sigma_{e}=\sqrt{\epsilon \beta+D^{2}\left(\frac{\Delta p}{p}\right)^{2}},
$$

where $\epsilon$ is the geometric emittance, $\beta$ the beta amplitude function, $D$ the dispersion, and $\Delta p / p$ is the fractional momentum spread of the beam. Although the horizontal and vertical dispersion at the LWIP are nominally zero, there will be a finite amount due to residual dispersion from beam misalignment in the extraction line quadrupoles or residual $x-y$ coupling. Dispersion and coupling are measured and corrected using the Flight Simulator software [14], which measures the electron beam trajectory using the high resolution CBPM system [15] as a function of electron beam energy. The energy is modulated by adjusting the damping ring rf frequency, and the calculated coupling and

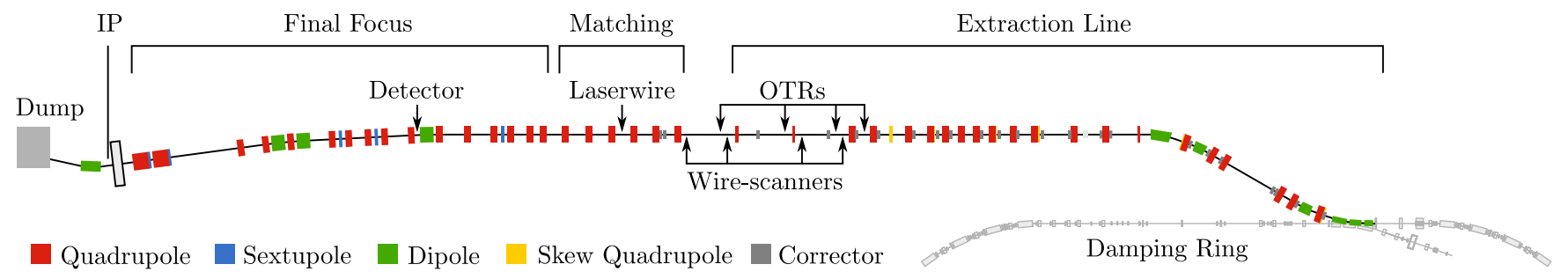

FIG. 1. Schematic of the ATF2 extraction line showing the location of the laserwire system as well as the detector located immediately behind the first dipole magnet after the laserwire interaction point. The wire scanners and OTR monitors are also shown at the end of the extraction line section. All quadrupoles and sextupoles in the matching and final focus sections are on individual three-axis mover systems. 


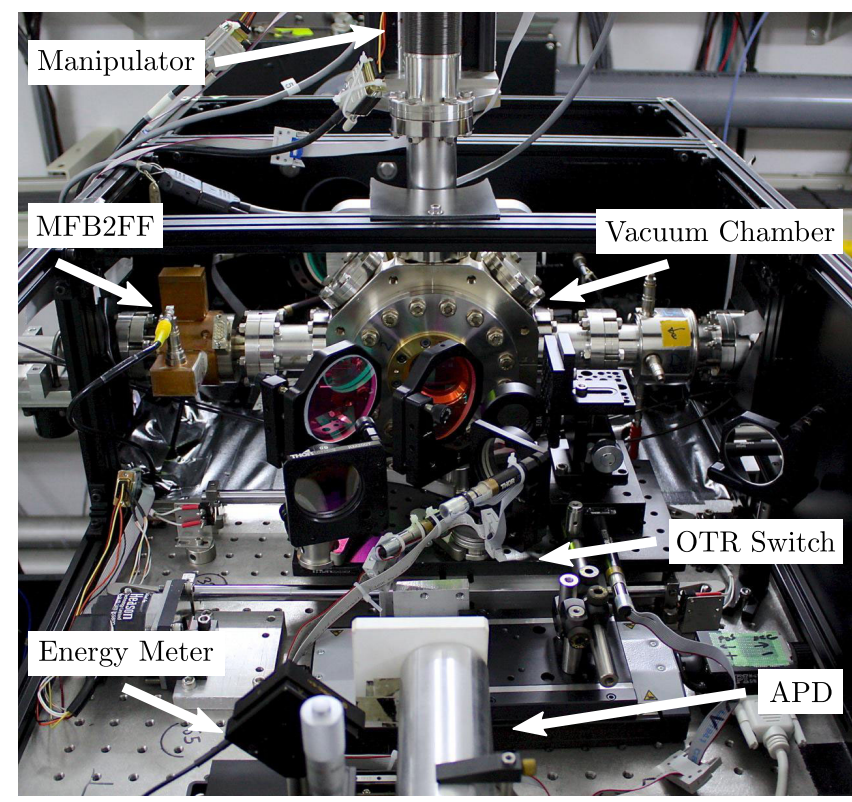

FIG. 2. Photograph of the laserwire installation in the ATF2 beam line. The electron beam travels from right to left and the laser beam enters behind the vacuum chamber and exits towards the reader. The manipulator for the OTR and alignment screen can be seen on top of the vacuum chamber. The avalanche photodiode (APD) used for timing and the laser pulse energy meter can be seen in the foreground. The high resolution CBPM MFB2FF is also shown attached to the laserwire vacuum chamber. The small optical breadboard (OTR switch) allows one to switch between the high power laser path for laserwire and the low intensity OTR path.

dispersion corrections applied using four upstream skew quadrupoles in combination.

The CBPM system provides high resolution position measurement at 45 locations through the extraction line, matching section, and final focus section of the ATF2. The majority of the CBPMs are mounted to the pole faces of the quadrupoles in the matching and final focus sections, with the remainder at other points in the extraction line. There are CBPMs in the quadrupoles before and after the LWIP; however, the CBPM afterwards is on the far side of the quadrupole, and so the trajectory cannot be treated as ballistic between the two. A high resolution CВРM,

TABLE I. ATF2 parameters.

\begin{tabular}{lccl}
\hline \hline Parameter & Symbol & Value & Units \\
\hline Beam energy & $\mathrm{E}$ & 1.30 & $\mathrm{GeV}$ \\
Horizontal emittance & $\gamma \epsilon_{x}$ & $4 \times 10^{-6}$ & $\mathrm{~m} \mathrm{rad}$ \\
Vertical emittance & $\gamma \epsilon_{y}$ & $4 \times 10^{-8}$ & $\mathrm{~m} \mathrm{rad}$ \\
Bunch repetition rate & $f_{\text {bunch }}$ & 3.12 & $\mathrm{~Hz}$ \\
Bunch length & $\sigma_{e z}$ & $\sim 30$ & $\mathrm{ps}$ \\
Electrons per bunch & $N_{e}$ & $0.5-10 \times 10^{9}$ & $\mathrm{e}^{-}$ \\
Fractional momentum spread & $\Delta p / p$ & 0.001 & \\
\hline \hline
\end{tabular}

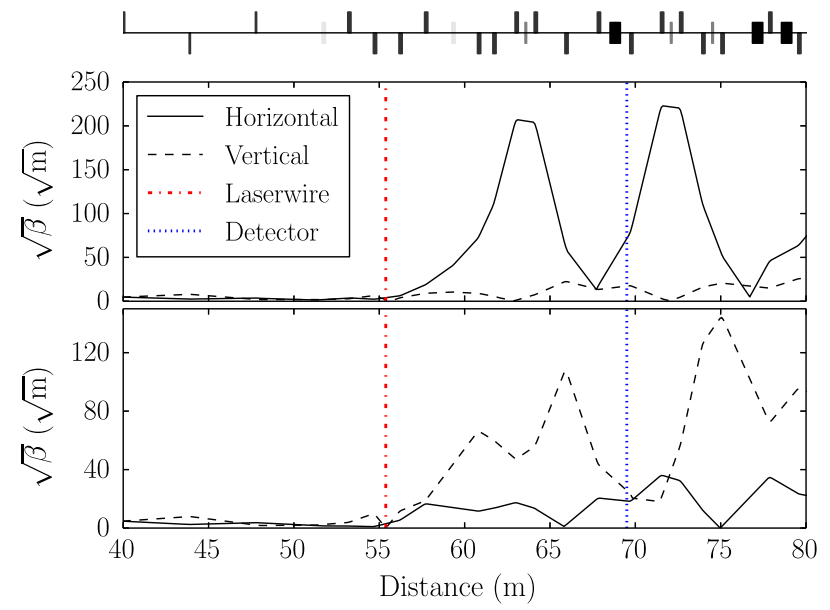

FIG. 3. Electron beam amplitude functions for the end of the extraction line, matching section and beginning of the final focus section. These are shown for normal ATF2 operation (top) and for laserwire operation (bottom). The laserwire and laserwire detector locations are shown by (red) dot-dashed and (blue) dotted vertical lines, respectively.

MFB2FF, is attached to the laserwire vacuum chamber and moves with it during laserwire scans. MFB2FF has a typical resolution of $70 \mathrm{~nm}$ at the bunch charge used during laserwire operations over a limited range of $<100 \mathrm{~nm}$ [15]. The scanning range of the laserwire exceeds this range and the mechanical offset and tilt of MFB2FF in relation to the laserwire vacuum chamber introduced $x-y$ coupling and degraded the resolution. Therefore, the electron beam position from MFB2FF was not suitable for spatial jitter subtraction during laserwire operation.

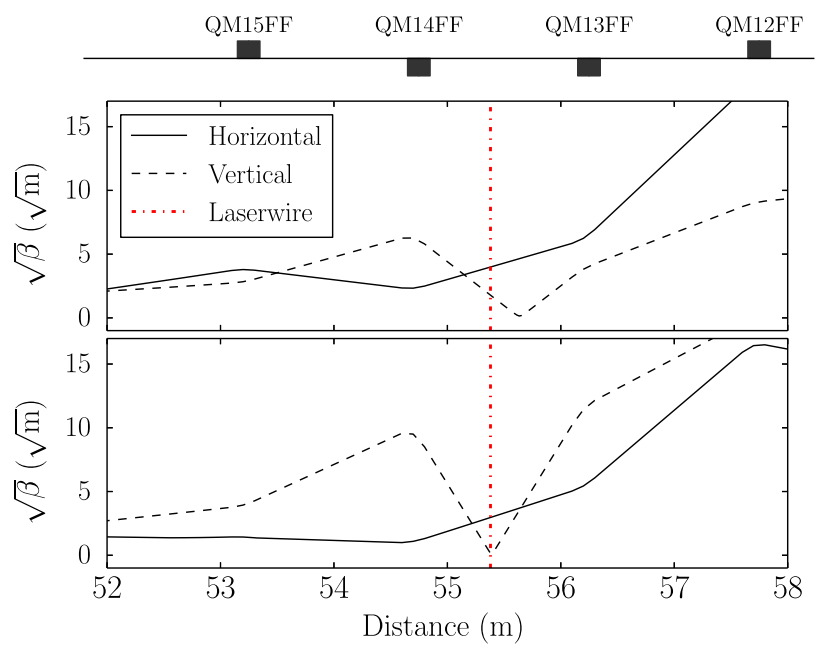

FIG. 4. Electron beam amplitude functions about the laserwire interaction point for normal ATF2 operation (top) where the vertical waist is located at the MFB2FF cavity BPM, and for laserwire operation (bottom), where the waist is moved to the laserwire location. 
Although the CBPMs near the LWIP could be used for spatial jitter subtraction at the LWIP, those around the laserwire are configured for a large dynamic range at the expense of resolution and have a typical resolution of $200 \mathrm{~nm}$. Given the approximately $1 \mu \mathrm{m}$ vertical electron beam sizes with the laserwire electron beam optics, spatial jitter subtraction was not possible. As the laserwire averages over multiple pulses, the approximate electron beam jitter of $\sim 0.2 \sigma$ at the ATF2 will contribute a systematic increase of approximately $1.9 \%$ to the measured electron beam size.

\section{B. Laserwire interaction}

A laserwire scan consists of the measurement of the Compton-scattered photon rate for different laser focus positions and is therefore the convolution of the transverse density functions of the laser beam and the electron beam in the axis of the scan. If both the electron and laser beams have Gaussian density functions, the convolution also has a
Gaussian form. However, unlike the wire in a traditional wire scanner, the laser beam size is not constant and expands on either side of the focus. The length scale of this is described by the Rayleigh range of the laser beam, which is the distance from the focus until the beam expands to twice its area. In the case where the electron beam size along the laser propagation axis is much smaller than the Rayleigh range, the laser width can safely be assumed to be constant across the electron beam width and the laserwire scan can be easily deconvolved independently of the horizontal size of the electron beam. However, in the case where the electron beam size is comparable to or greater than the Rayleigh range, the divergent laser beam interacts with the electron beam even when the laser focus is significantly displaced from the center of the electron beam as shown schematically in Fig. 5. To deconvolve the laserwire scan and measure the electron beam size, the form of the scan must be derived.

$$
N_{c}\left(\Delta_{x}, \Delta_{y}\right)=\frac{P_{l} N_{e} \lambda \sigma_{c}}{h c^{2}} \frac{1}{2 \pi \sigma_{e x}} \int_{-\infty}^{+\infty} \frac{1}{\sqrt{\sigma_{e y}^{2}+\sigma_{l}\left(x-\Delta_{x}\right)^{2}}} \exp \left\{-\frac{x^{2}}{2 \sigma_{e x}^{2}}-\frac{\Delta_{y}^{2}}{2\left[\sigma_{e y}^{2}+\sigma_{l}\left(x-\Delta_{x}\right)^{2}\right]}\right\} \mathrm{d} x .
$$

As the Compton cross section is constant for a given laser wavelength, electron beam, and collision geometry, the rate of Compton-scattered photons is determined by calculating the luminosity of the laser-electron beam collision for different offsets of the laser beam from the electron beam. The luminosity is calculated using the overlap integral between the four-dimensional density functions of the laser and electron beams. It was shown in [6] that by assuming Gaussian density distributions for the laser and electron beams and in the case where the laser pulse length is much longer than the electron bunch length, the laser distribution

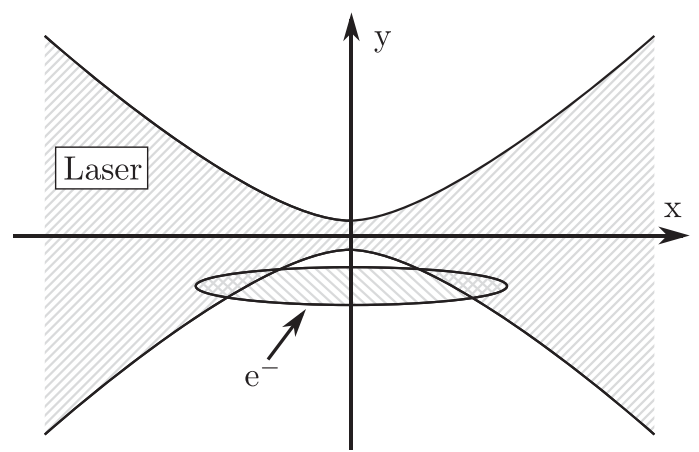

FIG. 5. Schematic of the laser focus showing its interaction with the high aspect ratio electron beam even when the laser focus is displaced from the electron beam. The vertical axis is expanded in scale compared to the horizontal axis to make the overlap clearer. can be treated as a static target and integration over three of the dimensions can be solved analytically yeilding Eq. (2). Here, $P_{l}, N_{e}, \lambda$, and $\sigma_{c}$ are the peak laser power, number of electrons per bunch, the wavelength of the laser, and the Compton cross section, respectively. This equation describes the number of Compton-scattered photons $N_{c}$ as a function of $\Delta_{x}$ and $\Delta_{y}$, the horizontal and vertical displacement of the laser focus from the center of the electron beam. The vertical and horizontal sizes of the electron beam are $\sigma_{e y}$ and $\sigma_{e x}$ and the vertical laser beam size is $\sigma_{l}\left(x-\Delta_{x}\right)$. The width of a laser beam is conventionally defined by $w$; however, we use $\sigma$ (where $w=2 \sigma$ ) for compatability with electron beam sizes. The propagation of a focused multimode laser beam is given by

$$
\sigma_{l}\left(x-\Delta_{x}\right)=\sigma_{l o} \sqrt{1+\left[\frac{\left(x-x_{\sigma l o}-\Delta_{x}\right)}{x_{R}}\right]^{2}},
$$

where $\sigma_{l o}$ is the minimum size of the laser at its focus and $x_{\sigma l o}$ is the location of the focus. The Rayleigh range $x_{R}$ is given by

$$
x_{R}=\frac{\pi\left(2 \sigma_{l o}\right)^{2}}{M^{2} \lambda},
$$

where $M^{2}$ is a linear scaling parameter describing the spatial quality of the laser beam with respect to one with a pefectly Gaussian transverse intensity profile and can be measured [16]. In the case where $\sigma_{e x} \ll x_{R}$, and the laser focus is aligned to the electron beam center $\left(\Delta_{x}=0\right)$, Eq. (2) simplifies to 


$$
\begin{aligned}
N_{c}\left(\Delta_{y}\right)= & \frac{P_{l} N_{e} \lambda \sigma_{c}}{h c^{2}} \frac{1}{(2 \pi)^{(3 / 2)} \sqrt{\sigma_{e y}^{2}+\sigma_{l o}^{2}}} \\
& \times \exp \left[-\frac{\Delta_{y}^{2}}{2\left(\sigma_{e y}^{2}+\sigma_{l o}^{2}\right)}\right],
\end{aligned}
$$

which has the form of a Gaussian. In this case, the laserwire vertical scan is independent of the horizontal beam size, and with knowledge of $\sigma_{l o}$, the $\sigma$ of the Gaussian laserwire scan can be analytically deconvolved to give the size of the electron beam, $\sigma_{e y}$.

However, in the case where $\sigma_{e x}$ is greater than or comparable to $x_{R}$, Eq. (2) must be used with the measured laser propagation and horizontal electron beam size $\sigma_{e x}$. This presents a significant limit on the use of a laserwire as a beam diagnostic and especially so at the ATF2 where the horizontal electron beam is expected to be $\sim 100 \mu \mathrm{m}$, which will be comparable to or greater than the Rayleigh range of the laser. The natural divergence of the laser beam cannot be avoided and is dictated by the wavelength used. The laserwire was operated with the assumption that horizontal measurements would be concurrently available from the OTR monitor installed at the LWIP [17].

The Compton-scattered photons from the laserwire have a broad spectrum with a maximum energy of

$$
h \nu_{\max }=E\left(\frac{2 \xi}{1+2 \xi}\right)
$$

where $E$ is the energy of the electron beam and $\xi$ the normalized photon energy in the electron rest frame $\left(\xi=\gamma h \nu / m_{e} c^{2}\right)$. In the case of the ATF2, with a laser wavelength of $532 \mathrm{~nm}$, the maximum Compton-scattered photon energy is $29.4 \mathrm{MeV}$. The Compton cross section is also dependent on the energy of the electron beam and the laser wavelength and in this case, the total cross section averaged over all scattered energies is $6.5 \times 10^{-24} \mathrm{~m}^{-2}$.

\section{Laser system and optical transport}

The laser system consists of a $Q$-switched neodymiumdoped yttrium aluminium garnet (Nd:YAG) amplifier seeded by a $357 \mathrm{MHz}$ mode-locked oscillator. The laser oscillator is stabilized and locked to an external signal generator at approximately $357 \mathrm{MHz}$ by means of a piezo actuator on one of the oscillator mirrors, with a typical temporal jitter of $<2$ ps. The signal generator is manually adjusted to match the ATF2 master oscillator frequency at the start of each operation period, as the ATF2 frequency is varied slightly according to the annual expansion cycle of the damping ring, and so the laserwire system must match it. A $10 \mathrm{MHz}$ reference signal relayed between the ATF2 master oscillator and the laserwire signal generator ensures a stable phase relationship between the laser pulses and the electron bunches. Two electro-optic modulators are used to isolate a single laser pulse from the oscillator, which is then amplified first in a regenerative amplifier followed by passage through a spatial filter and two single-pass linear amplifiers. The $1064 \mathrm{~nm}$ wavelength light is then frequency doubled in a beta-barium borate $\left(\beta-\mathrm{BaB}_{2} \mathrm{O}_{4}, \mathrm{BBO}\right)$ crystal providing $\sim 150 \mathrm{~mJ}$ pulses with a wavelength of $532 \mathrm{~nm}$ and a duration of $\sigma_{\tau}=70.8 \pm 0.6 \mathrm{ps}$ at $3.12 \mathrm{~Hz}$, the repetition rate of the ATF2.

The necessary trigger signals for the laser system are derived from the ATF2 damping ring extraction kicker thyratron charge and fire signals, which happen $1 \mathrm{~ms}$ before the extraction of the beam from the damping ring and the extraction itself, respectively. The trigger for the regenerative amplifier and linear amplifiers are independently controllable providing a large range of output pulse energy levels of the laser system. The locally generated $357 \mathrm{MHz}$ signal is first passed through a voltage controlled phase shifter allowing all of the laser trigger signals, that are created by multiple digital counter and delay generators, to be adjusted with respect to the electron beam arrival time while still maintaining their respective phase and timing relationships.

The laser system is mounted on an optical table in a temperature controlled lab on top of the accelerator concrete shielding blocks. A $10 \mathrm{~cm}$ diameter hole in both the table and the shielding blocks allows the laser beam to be transported in free space using mirrors in the accelerator environment. An automated mirror insert in the lab allows the laser beam path to be switched to a laser diagnostic line that consists of a series of relay mirrors providing exactly the same optical path length as to the laserwire lens beside the LWIP $(\sim 8.4 \mathrm{~m})$. A further mirror insert allows a $25 \mathrm{~mW}$ continuous-wave laser to be used for alignment purposes.

\section{Interaction point}

After the laser is transported to the laserwire interaction point, it is directed into a custom-made vacuum chamber with high damage threshold vacuum windows on either side to allow the laser beam to enter and exit. The vacuum chamber can be moved $\pm 3 \mathrm{~mm}$ in both the horizontal $x$ and vertical $y$ axes. A kinematic lens mount attached to the vacuum chamber is used to mount the laserwire lens, which allows precise control of the lens separation from the vacuum window as well as its angle. This is imperative as the vacuum window is an integral part of the lens optical design. The laserwire lens $(f=56.6 \mathrm{~mm})$ consists of two radiation-hard fused silica elements that are designed to correct geometric aberrations. The high radiation environment of the accelerator permits only fused silica to be used and, therefore, without different lens materials, chromatic aberrations cannot be corrected. The Nd:YAG laser source provides narrow bandwidth laser pulses that are easily accomodated by the $2 \mathrm{~nm}$ acceptance bandwidth of the lens negating any chromatic aberrations. By moving the vacuum chamber, the attached lens and therefore the laser focus also move. Optical position encoders provide $50 \mathrm{~nm}$ accuracy 


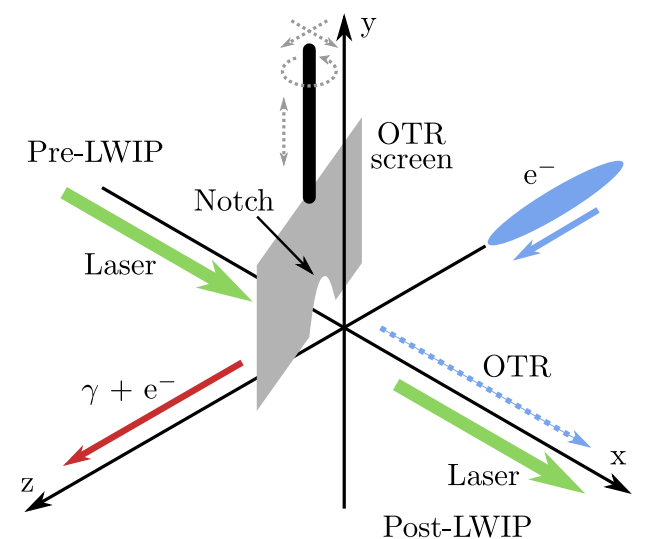

FIG. 6. Schematic of the beam geometry at the laserwire interaction point, including the OTR screen at $45^{\circ}$ to the electron beam direction, incoming electron bunch, outgoing electron bunch, OTR path, laser beam path, and Compton-scattered photons $(\gamma)$.

on the chamber position measurement. The coordinate axes of the interaction point are shown in Fig. 6.

A screen for both OTR and alignment is mounted on a vacuum manipulator arm that enters the vacuum chamber through the top access port. Manual micrometers allow the manipulator arm and therefore the screen to be moved in the $x$ and $z$ axes, while motorized actuators control the angle of the screen $\theta_{\text {OTR }}$ and its vertical position in the $y$ axis.

After the interaction point (post-LWIP), the laser beam exits the vacuum chamber through the vacuum window and is directed by two mirrors onto a laser energy meter. A plano-convex lens is used to bring the laser beam inside the active area of the energy meter. The post-LWIP optics are required to deal with the safe disposal of gigawatt peak power laser pulses, but also to image OTR, which is $\sim 10^{10}$ lower in intensity. To accomodate this, two separate switchable optical paths are used. Mirrors for each optical path are fixed on to a small optical breadboard that is mounted on top of a translation stage. Figure 7 shows the layout schematically.

An avalanche photodiode is used to simultaneously detect the laser light when strongly attenuated and a combination of OTR, optical diffraction radiation, and reflected synchrotron radiation [17] from the electron beam, allowing synchronization of both. The first postLWIP high reflectivity dielectic-coated mirror is used to attenuate the laser pulses without affecting the broadband OTR.

\section{E. Detector}

The laserwire detector is placed after the BH5X dipole magnet in the ATF2 lattice, which is the first bend after the LWIP and constitutes a bend of $2.927^{\circ}$. The box-shaped vacuum pipe in the dipole has an aluminum window $26 \mathrm{~mm}$

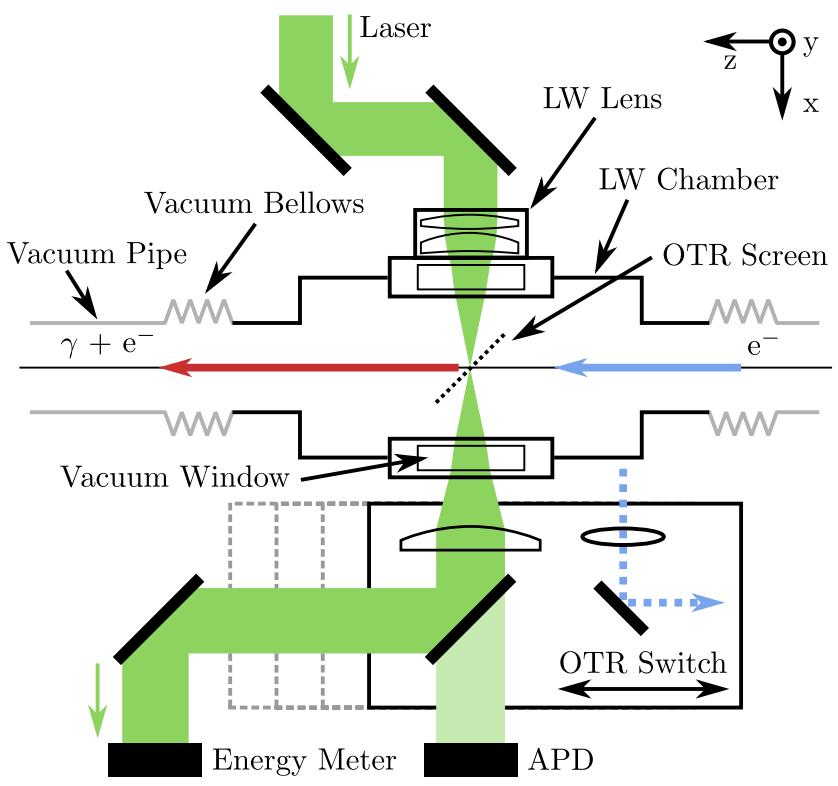

FIG. 7. Schematic of the laserwire (LW) interaction point in plain view showing the lens and vacuum windows attached to the vacuum chamber, the laser beam path (green), and post-LWIP optical switch for the OTR. The laser beam enters at the top of the diagram and is absorbed in the energy meter. The APD is used for timing purposes.

in diameter and $200 \mu \mathrm{m}$ in thickness at the end that allows the Compton-scattered photons from the laserwire to be detected.

The detector consists of a $4 \times 4 \times 0.6 \mathrm{~cm}^{3}(x \times y \times z)$ lead sheet that acts as a converter of photons to electronpositron pairs, followed by a $4 \times 4 \times 5 \mathrm{~cm}^{3}$ block of SP15 Aerogel. The Aerogel acts as a Cherenkov radiator for the electron-positron pairs and the Cherenkov light is guided in a light tight pipe, internally coated with aluminumized mylar, to a shielded photomultiplier tube out of the accelerator plane. The detector linearity was verified in [10]. Synchrotron radiation background was expected to be negligible as the synchrotron photon energy at the peak of its spectrum is $\sim 0.3 \mathrm{keV}$, which is insufficient to generate electron-positron pairs in the lead converter plate.

\section{F. Data acquisition system}

The data acquisition system is based around Experimental Physics and Industrial Control System (EPICS) database software [18]. This provides an easily extendable common interface level for all devices that are part of the experimental system as well as a graphical user interface using the Extensible Display Manager (EDM) and Python software for control, data storage, and data analysis. Individual devices are controlled through LabView or C software directly, which monitor command variables in the EPICS database and publish data and measurements to other variables. A suite of Python programs provides high level control of the laser system and laserwire experiment. 
TABLE II. Detector background levels before and during accelerator operation with an electron bunch population of $0.20 \pm 0.02 \times 10^{9} \mathrm{e}^{-}$.

\begin{tabular}{ll}
\hline \hline State & ADC counts \\
\hline ADC only & $406.7 \pm 2.9$ \\
Detector connected & $368.0 \pm 48.9$ \\
Detector powered & $366.7 \pm 49.0$ \\
Accelerator operation & $741.2 \pm 122.5$ \\
\hline \hline
\end{tabular}

Data is recorded from all devices for each machine cycle at $3.12 \mathrm{~Hz}$ as well as data from the CBPM system and other ATF2 beam instrumentation $[15,19]$.

\section{RESULTS}

The laserwire was operated in a series of experimental shifts during January and February 2013 and the results from these operation periods are presented in the following sections. The detector background level, laser propagation, and electron beam properties were all characterized before performing laserwire scans and are presented separately. The laser and electron beam alignment procedure developed is also detailed. The collision data and its analysis are then described in subsequent sections.

\section{A. Detector background}

The detector background level was measured both during and before accelerator operation. The value of the analogue to digital converter (ADC) was recorded with nothing attached to the ADC; when connected to the unpowered detector; when the detector was powered but there was no electron beam; and lastly during accelerator operations with no laser beam at the LWIP. These measurements represent the ADC pedestal and noise, the electrical pick up of the signal cables, the detector dark current, and the detector background level cumulatively and respectively. These results are summarized in Table II.

The ADC alone has a pedestal with a low level of noise. When connected to the laserwire detector, the pedestal is affected and the noise is significantly greater. The operation of the detector has a negligible increase in the measured noise. The electrical noise is most likely due to electrical pickup of the extraction kicker, which uses high voltage signals and can be readily observed on most electronics in the vicinity. This information was used to subtract the pedestal from the detector signal before each experimental shift.

\section{B. Laser characterization}

To accurately deconvolve the laserwire scans, precise knowledge of the laser beam propagation is required. This is accomplished by measuring the $M^{2}$ of the laser and the input laser beam profile to the laserwire lens, which can be used in combination to calculate the laser propagation at the LWIP. As the laser profile is affected by the passage through various components of the laser system, the slight change in alignment often necessary to maintain the required laser output power necessitates repeating the $M^{2}$ measurement. The laser diagnostic line was used to measure the $M^{2}$ and input beam profile in the lab without requiring access to the accelerator. Various reflective beam splitters were used in the diagnostic beam line to reduce the intensity to within the dynamic range of the laser beam profiler, allowing accurate measurement of the $M^{2}$ at the maximum output level of the laser system as is the case during laserwire operation.

Previous studies have shown that an input beam size of $4 \sigma=10-14 \mathrm{~mm}$ is required on the laserwire lens to produce the smallest possible focused spot size [10]. This size range allows the largest possible input beam size without incurring either diffraction effects due to the fixed aperture of the lens, or possible geometric aberrations. The input laser beam profile to the laserwire lens was measured at the end of the laser diagnostic line in the lab and an example profile is shown in Fig. 8.

The input beam size was adjusted using a demagnifying Gallilean telescope consisting of a plano-concave lens followed by a plano-convex lens that avoids ionization when the laser beam is focused in air. The telescope was placed close to the laser system aperture so that the telescope could be used to manipulate the input beam size without strongly affecting the laser divergence and therefore the $x$ location of the laser focus at the LWIP. Additionally, this arrangement allows the insertion mirror for the diagnostic line to be placed after any optics that affect the laser divergence, ensuring the laser beam at the end of the diagnostic line is exactly the same as at the entrance to the laserwire lens. A linear translation actuator was used to precisely adjust the spacing of the lenses, minutely adjusting the divergence and therefore the input laser beam size at the laserwire lens. The $4 \sigma$ laser beam widths (compliant with the ISO 11145-2 [20] standard) are shown in Fig. 9. As the laser was found to be astigmatic and

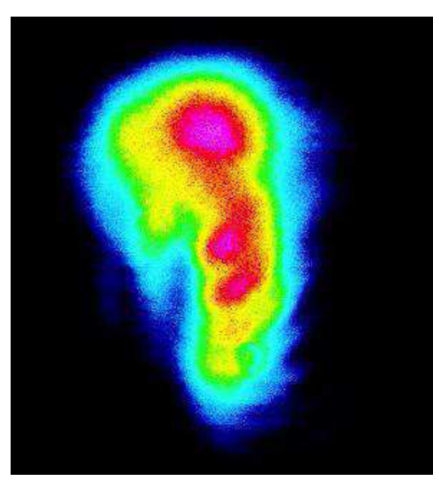

FIG. 8. Input laser profile measured at the end of the laser diagnostic line with telescope actuator at $12.5 \mathrm{~mm}$. 


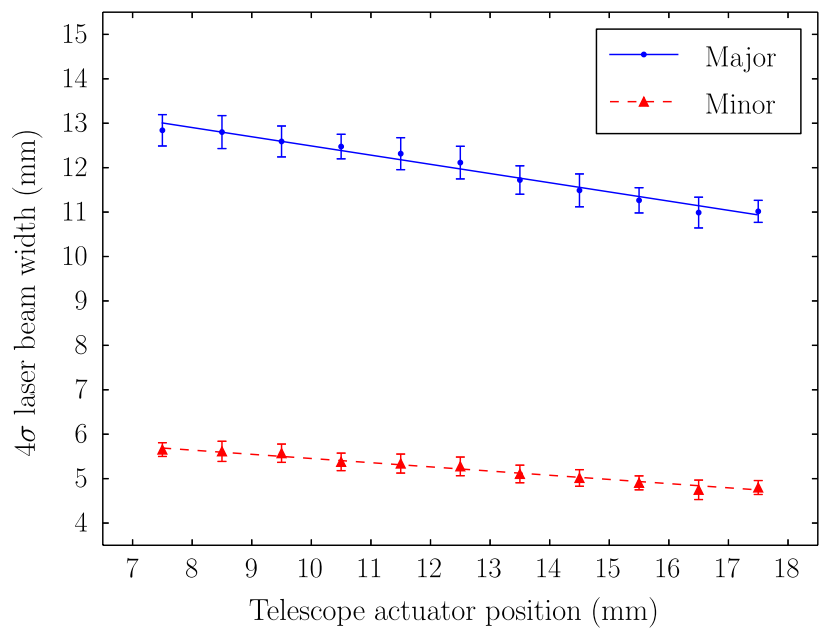

FIG. 9. $4 \sigma$ widths of the major and minor axes of the input laser beam profile as measured at the end of the laser diagnostic line as a function of telescope actuator position.

elliptical, the major and minor beam widths of the beam ellipse are shown. The telescope actuator was set at $12.5 \mathrm{~mm}$ for the laserwire operation period.

The $M^{2}$ of the laser was measured by placing a $f=$ $1.677 \mathrm{~m}$ (at $\lambda=532 \mathrm{~nm}$ ) plano-convex lens at the end of the laser diagnostic line to create a larger focused spot size over a greater distance. Profiles of the laser beam were recorded at various positions throughout the focus. The $4 \sigma$ widths along the intrinsic laser beam axes are shown in Fig. 10 along with a fit to the $M^{2}$ model [Eq. (3) with $\Delta_{x}=0$ ].

This shows that the laser is astigmatic with different focused spot sizes at different locations with different divergences. The intrinsic axes of the laser were found

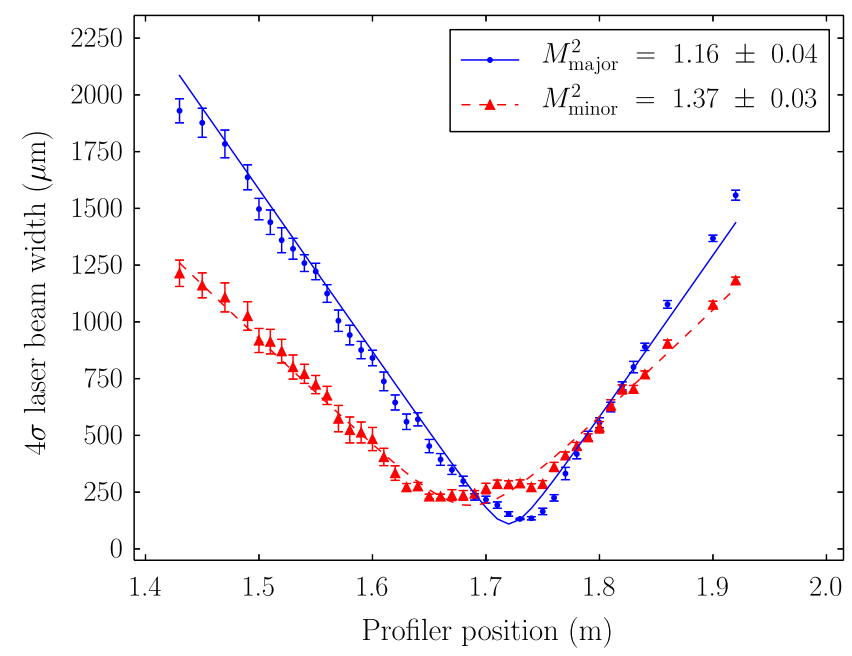

FIG. 10. Measured $4 \sigma$ widths of the laser beam through the focus created with a $f=1.677 \mathrm{~m}$ lens. The $M^{2}$ model is shown for each intrinsic axis of the laser propagation, which were found to be rotated to the extrinsic lab axes by $-17.4^{\circ}$.

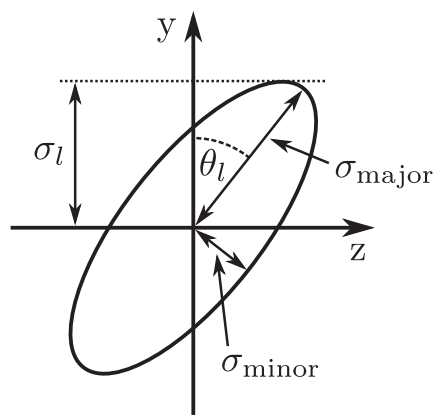

FIG. 11. Maximum extent of an ellipse described by the major and minor axes $\sigma_{\text {major }}$ and $\sigma_{\text {minor }}$, respectively, here representing $\sigma_{y, z}$ of the bivariate Gaussian laser photon distribution.

to be rotated to the (extrinsic) lab axes by $-17.4^{\circ}$. To deconvolve the laserwire scan, it is the distribution of photons in the vertical $(y)$ axis that is required. To calculate this, the laser is assumed to be a bivarate Gaussian as described by $\sigma_{z, y}$. As the projection of a bivariate Gaussian distribution is also Gaussian, the relevant vertical projection is the maximum extent of the ellipse, $\sigma_{l}$, depicted in Fig. 11 and described by

$$
\sigma_{l}=\sqrt{\left(\sigma_{l z} \sin \theta_{l}\right)^{2}+\left(\sigma_{l y} \cos \theta_{l}\right)^{2}}
$$

where $\theta_{l}$ is the angle of the laser axes with respect to the lab frame and the subscripts $z$ and $y$ denote the laser axis closest to that dimension in the lab frame. Here, the major axis is closest to the $y$ dimension. The laser propagation parameters $\sigma_{o}$ and $x_{\sigma o}$ in each axis were scaled to the LWIP using the ratio of the focal lengths of the $M^{2}$ measurement lens and the laserwire lens. Each axis is described by Eq. (3) using the scaled parameters, and the projected vertical size was calculated using Eq. (7) as shown in Fig. 12. The laser propagation was measured each week after maintenance was carried out on the laser system, and the relevant measurement was used in the analysis of the laserwire data. In the case of the laserwire data presented here, the minimum vertically projected laser spot size was $\sigma_{l}=1.006 \pm 0.032 \mu \mathrm{m}$.

The laser pointing stability was measured at the end of the laser diagnostic line to estimate the pointing stability of the laser at the LWIP by recording 600 laser beam profiles and the centroid of each calculated. The standard deviation of the centroids in the horizontal and vertical was measured to be 125.7 and $132.7 \mu \mathrm{m}$, respectively, at the laserwire lens. This measured spatial variation can be scaled by the beam size at the ratio of the input laser beam size to that at the LWIP to give a laser position variation of $\sim 40 \mathrm{~nm}$ in both dimensions. This spatial variation therefore systematically increases the measured electron beam size by approximately $0.08 \%$, which was deemed to be a negligible contribution and therefore not subtracted from the laserwire scans. 


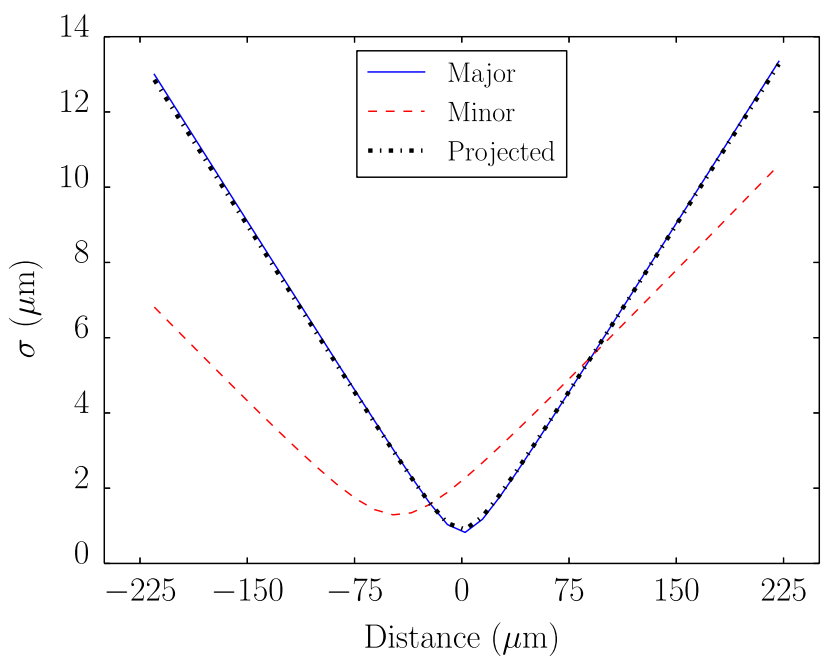

FIG. 12. Calculated projected vertical sigma for the laser as well as the two axes of propagation at the LWIP. The distance is zeroed about the minimum of the projected vertical size where the laser is most intense and the Cherenkov signal greatest.

\section{Electron beam characterization}

At the start of laserwire operations, the laserwire electron beam optics were set and the trajectory of the electron beam adjusted to quadrupole centers. After this, the dispersion and coupling were measured and corrected using the Flight Simulator software by changing the damping ring frequency in $1 \mathrm{kHz}$ steps over a range of $5 \mathrm{kHz}$. This was repeated several times to accurately correct coupling and dispersion. The measured residual dispersion at the LWIP was $D(x)=4.215 \pm 0.515 \mathrm{~mm}$ and $D(y)=0.095 \pm 0.023 \mathrm{~mm}$. This is acceptable and should make a negligible contribution to the vertical electron beam size, given the energy spread of the electron beam at the ATF2.

The emittance of the extracted electron beam in the ATF2 can be measured either using wire scanners or the multi-OTR system (mOTR) [19]. Measuring the emittance using the wire scanners during laserwire operations is impractical due to time constraints. During early 2013, the mOTR system was being upgraded and was not available for use during laserwire operations.

\section{Alignment}

To achieve collisions between the laser and electron beams, they must be spatially and temporally overlapped. Both of these functions were achieved using the OTR screen as an alignment tool.

\section{Laser alignment}

Before operations, the laser beam must be precisely aligned to the center of the laserwire lens as well as perpendicularly to the vacuum window and lens assembly to ensure the diffraction limited focused spot size is achieved. The low power alignment laser was first used without the laserwire lens. The two mirrors before the LWIP were adjusted such that the back reflection from the vacuum window overlapped with the incoming laser beam back to its source. A mounted mirror was then placed in the kinematic laserwire lens mount and the angle of the mount adjusted until the reflected laser beam also overlapped the incoming laser beam. This ensured the lens and window were parallel to each other and that no optical aberrations were introduced, as these would increase the focused spot size. The alignment was verified with the main laser beam at low power. After this procedure, the mirror was removed from the lens mount and the laserwire lens was replaced.

\section{Spatial alignment}

During access periods before operation, the laser was operated at low pulse energy and attenuated heavily so as not to cause damage to the OTR screen. The OTR screen was moved vertically to find the point where it intercepted the laser focus as observed in the post-LWIP optical system. The manual micrometers were adjusted to position the OTR screen along the $x$ axis so that the vertical distance required to occlude the laser beam was minimized, ensuring that it was centerd at the laser focus in the $x$ dimension. During experimental shifts, with the OTR screen set to the vertical reference position, the laserwire vacuum chamber was then scanned vertically until the electron beam was intercepted (the OTR screen arm moves with the chamber). When the screen intercepts the electron beam brehmstraahlung radiation is produced that is detected by the wire scanner detector behind the laserwire detector. The chamber was aligned to the point where half the maximum

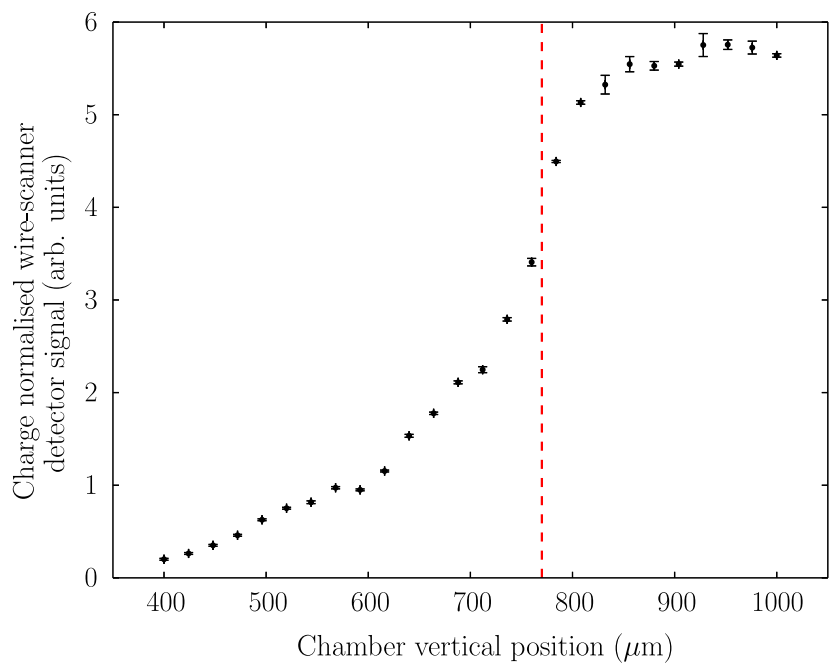

FIG. 13. Measured brehmstraahlung radiation as a function of vertical chamber position with the OTR screen at the laser focus reference position. The red dashed line shows the chosen alignment position. 


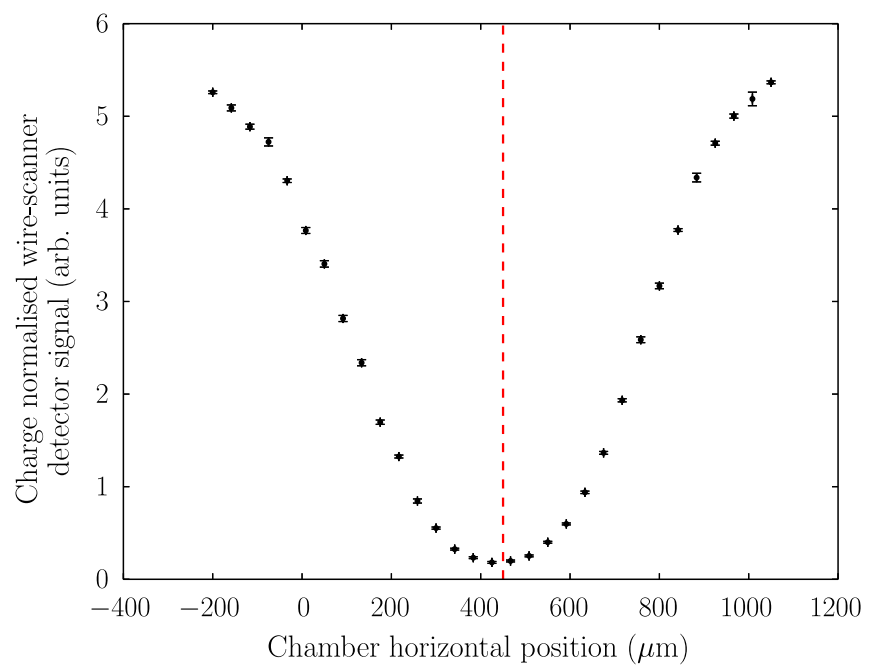

FIG. 14. Measured brehmstraahlung rate radiation as a function of horizontal chamber position with the OTR screen at the laser focus reference position. The red dashed line shows the chosen alignment position.

brehmstraahlung radiation was produced as shown in Fig. 13.

During operations in 2011, the OTR screen was accidentally damaged by the high energy pulsed laser beam creating a semicircular hole at the bottom of the screen approximately $500 \mu \mathrm{m}$ in diameter. This notch proved to be extremely useful as it allowed horizontal alignment of the laser beam for the first time. The OTR screen was placed approximately $200 \mu \mathrm{m}$ above the electron beam as found with vertical alignment and then the chamber was scanned in the horizontal $x$ axis. A minimum in brehmstraahlung radiation indicated the passage of the electron beam through the notch in the screen, which in turn indicates alignment to the laser focus. An example of this horizontal alignment scan is shown in Fig. 14.

\section{Temporal alignment}

To perform temporal alignment, the OTR screen was raised above the laser focus reference position to allow laser light to pass through the LWIP to the APD. The vacuum chamber was then lowered until OTR was produced by the screen. The APD signal was observed on a $1 \mathrm{GHz}$ bandwidth, 5 gigasamples $\mathrm{s}^{-1}$ oscilloscope. Because of bandwidth limitations of the APD, cables, and the oscilloscope, both the laser and OTR pulses are represented by approximately $1 \mathrm{~ns}$ pulses on the oscilloscope used. However, it is still possible to perform the temporal alignment by attenuating the laser light to match the OTR signal level and adjusting the laser system timing until the APD signal is doubled. This method allowed alignment within $40 \mathrm{ps}$, which was sufficient to attain detectable collisions.

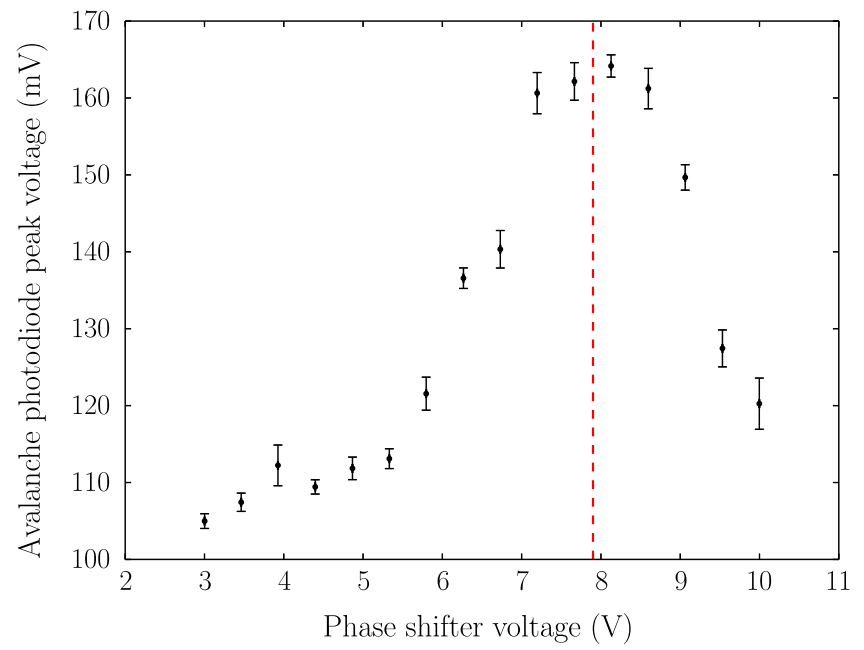

FIG. 15. Peak APD signal as a function of laser phase controlled by a $0-10 \mathrm{~V}$ static voltage. The red dashed line shows the chosen phase setting.

The laser timing was intially adjusted in integer (357 MHz, $2.8 \mathrm{~ns}$ ) clock cycles to align the laser and OTR signals as close as possible. The voltage-controlled phase shifter was then used to adjust the phase between the electron beam and the laser pulses for precise alignment. The maximum voltage of the oscilloscope trace was recorded as a function of phase shifter voltage as shown in Fig. 15, to ascertain the best timing overlap. Given the laser pulse and electron bunch lengths, the laser timing jitter of $<2$ ps as well as the electron bunch timing jitter of $<5$ ps will increase the jitter of the detected laserwire signal level by $\ll 1 \%$, but it will not affect the measured electron beam size.

A summary of the alignment accuracies in comparison to the nominal sizes in each dimension is given in Table III. This alignment procedure regularly led to detectable collisions allowing optimization of the alignment.

\section{E. Collision data}

\section{Initial collisions}

Once detectable collisions were established, the alignment between the laser beam and the electron beam was optimized to produce the maximum number of Comptonscattered photons by scanning the laserwire vertically, then

TABLE III. Summary of the typical alignment accuracy $\Delta$ and the percentage of the nominal approximate size $\sigma$ in each dimension.

\begin{tabular}{lccc}
\hline \hline Dimension & $\Delta$ & $\sigma$ & $\%$ Error \\
\hline Vertical & $5 \mu \mathrm{m}$ & $1 \mu \mathrm{m}$ & 500 \\
Phase & $40 \mathrm{ps}$ & $70 \mathrm{ps}$ & 57 \\
Horizontal & $50 \mu \mathrm{m}$ & $120 \mu \mathrm{m}$ & 41 \\
\hline \hline
\end{tabular}




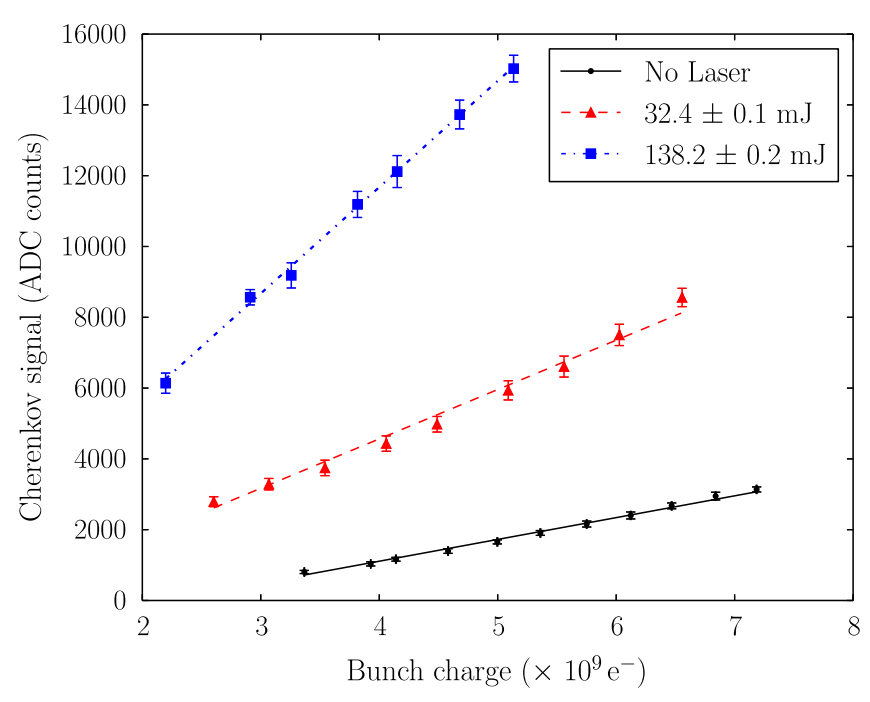

FIG. 16. Variation of the Cherenkov signal with electron bunch charge for various laser levels.

in phase, and then horizontally. This sequence of scans was repeated until no further improvement was observed.

During operations it was immediately clear that the vertical laserwire scans had a non-Gaussian shape with a narrow peak and broad wings observable up to $30 \mu \mathrm{m}$ away from the peak of the scan. To accurately sample this shape in the minimum time, scans with variable step sizes were used, which were approximately distributed according to a cubic polynomial - what we here call a nonlinear scan. As a large number of steps are required in the center of the scan, the center of the scan must be within $\sim 1 \mu \mathrm{m}$ of the peak of the measured signal for the best sampling. Therefore, an initial vertical scan with a low number of samples and linear step sizes was used for centering purposes. Furthermore, due to the non-Gaussian shape, Eq. (2) must be used. This requires knowledge of the horizontal electron beam size. To both optimize the alignment and measure the horizontal electron beam size, the laser focus was scanned horizontally across the electron beam over a $3 \mathrm{~mm}$ range. After this, the chamber was positioned at the center of the horizontal scan and a detailed nonlinear vertical scan was performed.

\section{Signal linearity}

With the Compton signal maximized, the electron bunch charge and laser pulse energy were varied independently to ascertain the signal correlations and linearity. The bunch charge was varied by modulating the accelerator laser photocathode pulse energy. The delivered bunch charge to the extraction line varies very nonlinearly with photocathode laser pulse energy, but the settings were chosen to give approximately linear steps. This charge ramp was repeated with no laser at the LWIP, as well as medium and maximum laser output power levels as shown in Fig. 16.

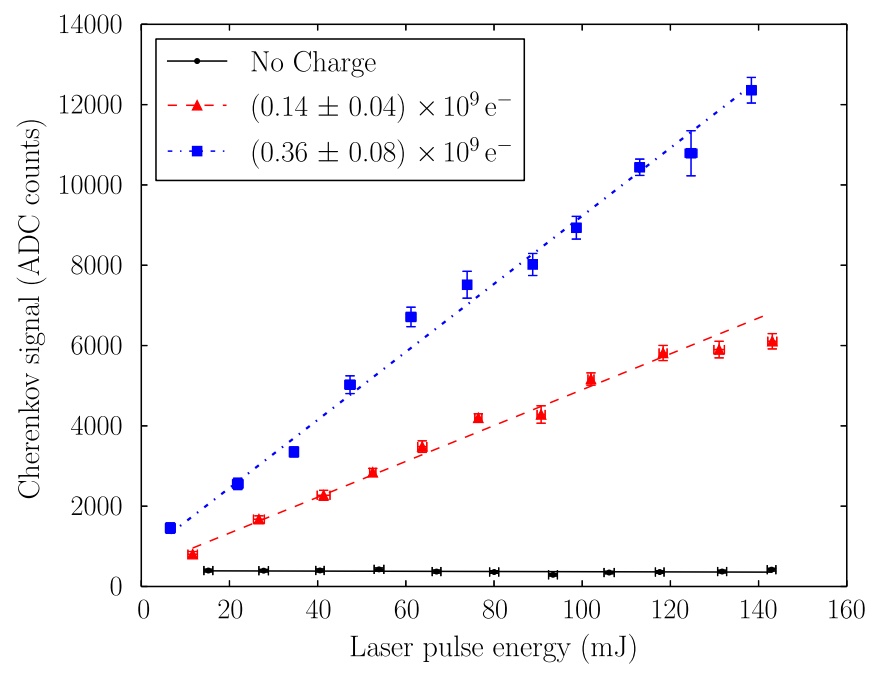

FIG. 17. Variation of the Cherenkov signal with laser pulse energy for various electron bunch charges.

This shows a linear dependence with charge in all cases. The charge ramp was not continued to the highest bunch charge with the highest laser output to avoid detector saturation during the measurement. After this, complementary scans were performed by ramping the laser pulse energy while keeping the bunch charge fixed as shown in Fig. 17.

In this case, despite showing an approximately linear relationship with laser pulse energy as expected, a greater degree of variation was seen. Here nonlinearities are most likely due to the variation of laser profile with laser pulse energy, which due to the laser technology employed is not expected to be consistent. The laser characterization was carried out at the laser pulse energy that would be used for operations.

These linearity scans encompass a much larger variation of both electron bunch charge and laser pulse energy than will be encountered during a scan and so from this data it can be seen that the detector will be linear over the small range of varation of bunch charge and laser pulse energy during a laserwire scan.

\section{Combined horizontal and vertical analysis}

The horizontal scans were initially fitted using a Gaussian model as this allows independent analysis of the horizontal and vertical scans. However, the horizontal scan is the convolution of the laser intensity in the $x$ axis with the Gaussian distribution of the electrons in the same dimension. In the case where the Rayleigh range is much less than the electron beam size $\left(x_{R} \ll \sigma_{e x}\right)$ the convolution is dominated by the electron beam shape and the Gaussian fit is acceptably accurate. Although the Gaussian model was found to provide an accurate fit in previous operations [10], the horizontal scan data deviated from the Gaussian model curve noticeably. Therefore, the integral in Eq. (2) 


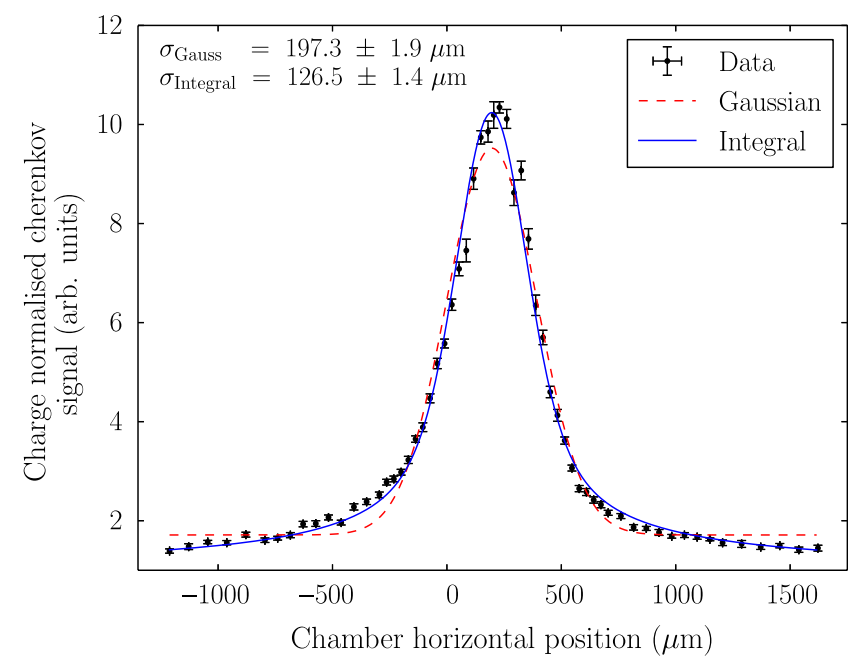

FIG. 18. Comparison of Gaussian and overlap integral models for the horizontal laserwire scan.

was used to fit a pair of horizontal and vertical scans simultaneously to determine both $\sigma_{e x}$ and $\sigma_{e y}$. The horizontal scan is shown in Fig. 18 with both the Gaussian and overlap integral models for comparison.

Importantly, the extracted horizontal size is considerably different from that found using the Gaussian model, which if incorrectly used to deconvolve the vertical laserwire scans yields an inaccurate vertical electron beam size. It had originally been envisioned that a single horizontal scan could be used to deconvolve all the vertical laserwire scans for a given measurement period (such as an 8-hour experimental shift). Even with adjustments made to the vertical beam size that would affect the horizontal size, the deconvolution was expected to be relatively insensitive to the horizontal size. However, even with changes in horizontal size of a few percent, this proved to be untenable and

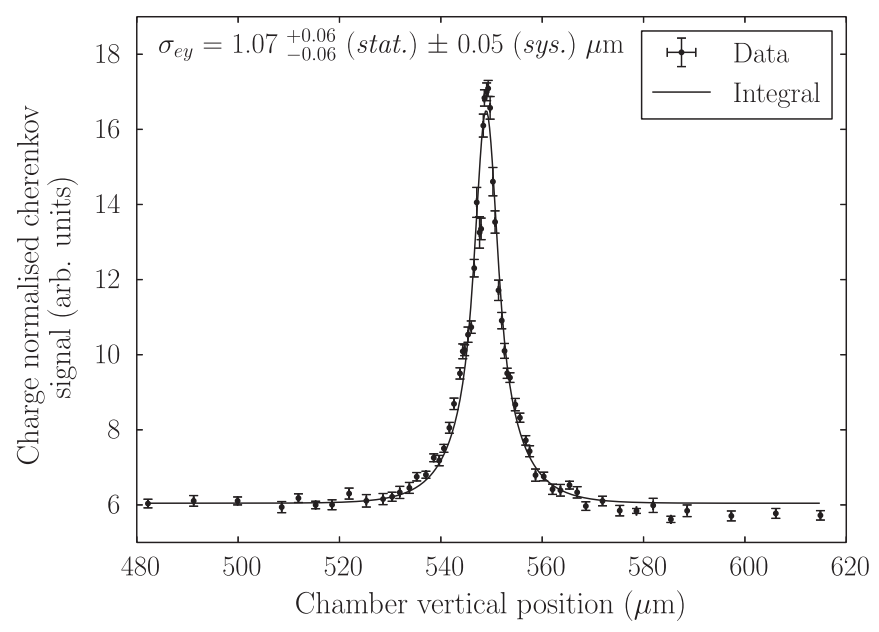

FIG. 19. Nonlinear step size laserwire scan with the smallest measured electron beam size. so horizontal and vertical scans were made each time for a complete measurement.

\section{Smallest vertical scan}

The electron beam optics were manipulated to minimize the electron beam size at the LWIP as measured by the laserwire. The laserwire scans shown in Fig. 19 and Fig. 20 are the vertical and horizontal laserwire scans, respectively, that were analyzed together and constitute the smallest vertical electron beam profile measured. These were recorded with an electron bunch population of $0.51 \pm$ $0.05 \times 10^{10} \mathrm{e}^{-}$.

The measured vertical electron beam size was $1.07_{-0.06}^{+0.06}$ (stat) \pm 0.05 (sys) $\mu \mathrm{m}$ and the horizontal beam size was $119.0_{-2.4}^{+2.4}$ (stat) \pm 0.01 (sys) $\mu \mathrm{m}$. The analysis was performed using Minuit minimization software using a weighted least squares method that allowed for asymmetrical uncertainties using the Minos algorithm [21]. The systematic uncertainties were found by calculating the standard deviation of the fit parameters from randomly sampling the laser parameters from the $M^{2}$ model analysis with their associated uncertainties. The calculated laserwire signal from the fit as a function of vertical and horizontal chamber positions is shown in Fig. 21. This shows that the vertical scan reaches a lower signal level than the horizontal scan at the edges of the scan, which can also be seen in Figs. 19 and 20.

\section{Quadrupole scan}

The laserwire was used to profile the electron beam throughout a quadrupole scan of the vertically focusing quadrupole immediately before the LWIP, QM14FF. The magnet current was varied from $-80 \mathrm{~A}$ to $-104 \mathrm{~A}$ in $3 \mathrm{~A}$ steps. At each point, a short range, low sample number vertical scan was performed to vertically center the laser

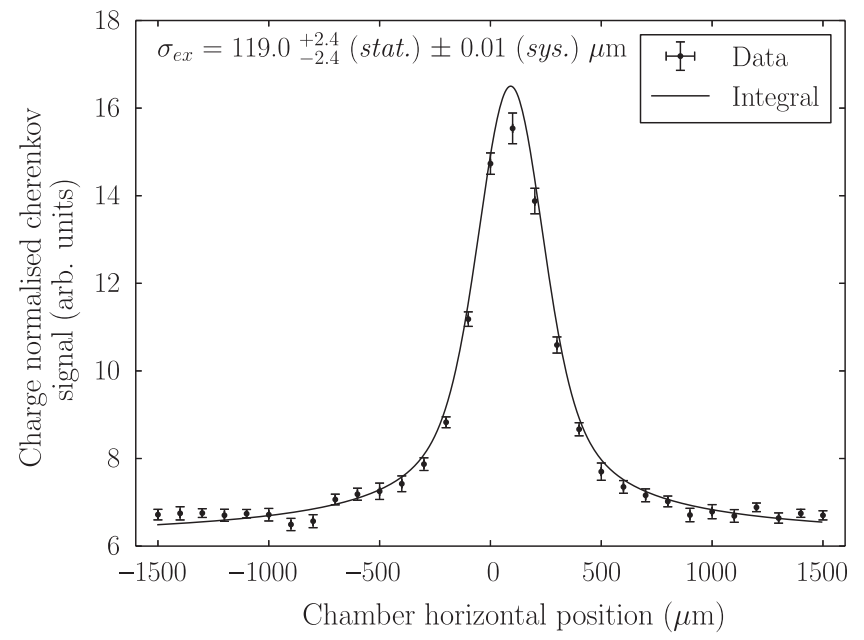

FIG. 20. The corresponding horizontal laserwire scan for the smallest vertical scan, which was required for the combined analysis. 


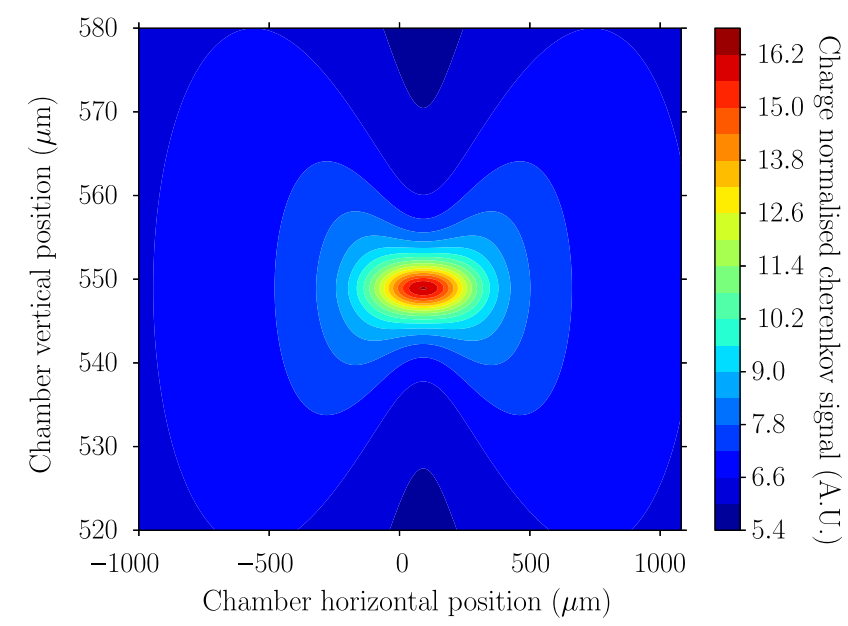

FIG. 21. Calculated laserwire signal for a range of horizontal and vertical chamber positions from the simultaneous fit of the horizontal and vertical laserwire scans. The scans were performed immediately after each other ensuring consistent experimental conditions.

beam. After this, a detailed horizontal scan was performed followed by a nonlinear vertical scan. The horizontal and nonlinear vertical scans were fitted together to yield $\sigma_{e x}$ and $\sigma_{e y}$ as shown in Fig. 22.

The vertical sizes show a clear hyperbolic focus as expected with a maximum measured size of $7.30_{-0.15}^{+0.16}$ (stat) \pm 0.17 (sys) $\mu \mathrm{m}$ and a smallest size of $1.43_{-0.05}^{+0.05}$ (stat) \pm 0.04 (sys) $\mu \mathrm{m}$. The horizontal sizes show a nearly linear progression as expected. The scan shows there is still clear variation in the measured scan size at $\sim 1.5 \mu \mathrm{m}$ with no flattening at the minimum of the scan, indicating the resolution limit of the laserwire has not been reached. The horizontal scan shows a greater degree of

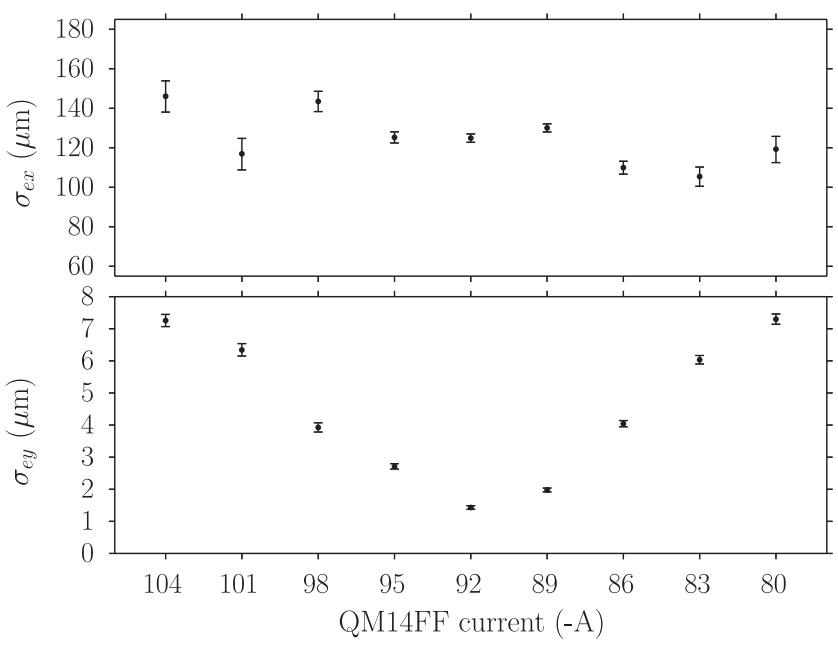

FIG. 22. Horizontal (top) and vertical (bottom) electron beam sizes measured by the laserwire using combined analysis for various currents of QM14FF, the vertical focusing quadrupole immediately before the LWIP.

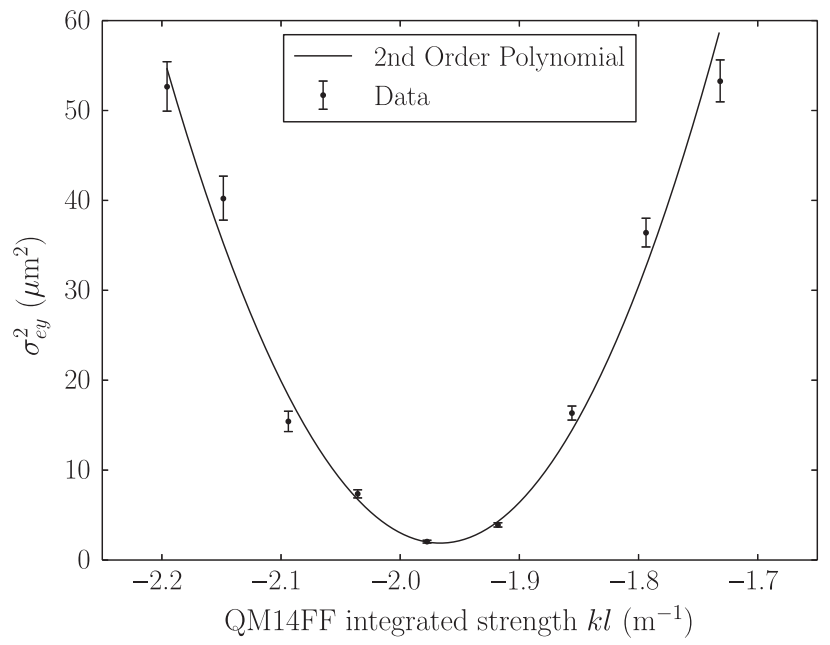

FIG. 23. Measured electron beam size squared as a function of QM14FF strength. The least squares fit to a parabola for the emittance extraction is also shown.

variation from the expected linear shape, which is due to variation in the electron beam energy over the time of the scans. The set of three laserwire scans required to make a measurement of the electron beam takes approximately $20 \mathrm{~min}$ and the whole quadrupole scan $\sim 3.5 \mathrm{hr}$. A low electron bunch population of $0.2 \times 10^{10} \mathrm{e}^{-}$was used in this case to provide the most stable condition over the duration of the measurement.

Figure 23 shows the measured vertical sizes squared as a function of QM14FF integrated quadrupole strength $\mathrm{kl}$, which is modeled using the thick lens formalisim [22]

$$
\sigma_{e y}^{2}=a\left[m_{11}(k l)+b m_{12}(k l)\right]^{2}+c m_{12}^{2}(k l),
$$

where $a, b$, and $c$ are free parameters. $m_{11}$ and $m_{12}$ are given by

$$
\begin{gathered}
m_{11}(k l)=S_{11} \cos (\sqrt{k} l)-S_{12} \sqrt{k} \sin (\sqrt{k} l), \\
m_{12}(k l)=S_{11} \frac{1}{\sqrt{k}} \sin (\sqrt{k} l)-S_{12} \cos (\sqrt{k} l),
\end{gathered}
$$

where $S$ is the transfer matrix between the quadrupole and the measurement plane. The geometric emittance is given by

$$
\epsilon=\sqrt{a c} .
$$

There is only a drift segment of the beam line between the QM14FF quadrupole and the LWIP, so $S_{12}$ is the drift distance, $692.66 \pm 1.00 \mathrm{~mm}$ and $S_{11}$ is 1 . From the fit to Eq. (8), $a$ and $c$ were found to be $2026.05 \pm 47.40 \times$ $10^{-12} \mathrm{~m}^{2}$ and $3.365 \pm 0.235 \times 10^{-12}$, respectively. Using these values, the measured projected geometric emittance is 


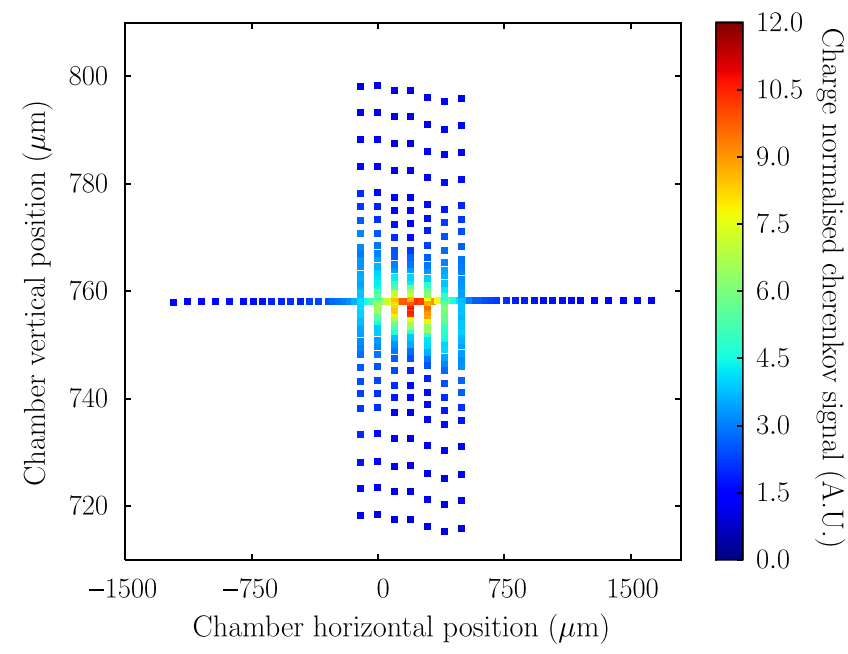

FIG. 24. Charge normalized Cherenkov signal sampled in multiple vertical laserwire scans at different horizontal positions. A long range horizontal scan is also included.

$82.56 \pm 3.04 \mathrm{pm}$ rad. This value is a relatively large emittance compared to the typical optimized vertical value of the ATF2, which in the damping ring is $10 \mathrm{pm}$ and $10-30 \mathrm{pm}$ in the extraction line. The extraction line optics optimization procedure [13] was not carried out in full for laserwire operation periods and higher emittance values are to be expected.

\section{Detailed vertical slicing}

To fully map the laser-electron collisions, a detailed set of nonlinear vertical laserwire scans was recorded at several horizontal locations as well as a long range horizontal scan. All of the data were fitted simultaneously using the overlap integral model. The signal levels at each location are shown in Fig. 24, and the parameters from the fit are given in Table IV. In this case, the higher number of laser focus positions reduces the statistical uncertainty of the fit to the model significantly and the systematic uncertainties now dominate.

Given the high aspect ratio of the electron beam, only a small amount of $x-y$ coupling would cause the measured

TABLE IV. Parameters from fitting multiple laserwire scans. The first uncertainty is the asymmetric statistical uncertainty from the fit and the second is the associated systematic uncertainty.

\begin{tabular}{lrll}
\hline \hline Parameter & Fit value & $\pm($ stat $)$ & $\pm($ sys $)$ \\
\hline Amplitude & 9.609 & $\pm_{0.041}^{0.042}$ & \pm 0.049 \\
$x_{0}$ & 195.728 & $\pm_{0.525}^{0.525}$ & \pm 0.008 \\
$y_{0}$ & 756.980 & $\pm_{0.016}^{0.016}$ & \pm 0.002 \\
Background & 1.045 & $\pm_{0.005}^{0.005}$ & \pm 0.021 \\
$\sigma_{e x}$ & 120.588 & $\pm_{0.650}^{0.651}$ & \pm 2.339 \\
$\sigma_{e y}$ & 1.707 & $\pm_{0.023}^{0.023}$ & \pm 0.051 \\
\hline \hline
\end{tabular}

vertical projection of the electron beam to be significantly larger than the intrinsic vertical size of the electron beam. Furthermore, there is the possibility that the laser beam could be at a small angle with respect to the electron beam, which, even if no coupling were present in the electron beam, would result in a larger measured beam size. Unlike the two scan analysis performed already, the larger $x-y$ area covered by the data points allows the roll of the electron beam to be analyzed. This would conventionally be determined by applying a rotation of coordinates to the model and allowing the angle $\theta_{e}$ to be a free variable in the minimization. However, this would prevent an analytical solution to three dimensions of the overlap integral, requiring a numerical solution for three dimensions instead of one. Although possible, this would significantly complicate the data analysis and reduce the accuracy. Alternatively, if a small angle of rotation is assumed, the data can be rotated instead of the model achieving approximately the same result. This can be safely assumed due to the high aspect ratio of the electron beam as a small rotation of the data will not significantly affect the projection in the horizontal $(x)$ axis, whereas it will significantly affect the projection in the vertical $(y)$ axis. If the electron beam is rotated with respect to the laserwire, the projection in the $y$ dimension, $\sigma_{e y^{\prime}}$ will be measured and is described by Eq. (12):

$$
\sigma_{e y^{\prime}}=\sqrt{\left(\sigma_{e y} \sin \theta_{e}\right)^{2}+\left(\sigma_{e x} \cos \theta_{e}\right)^{2}} .
$$

As the angle of rotation $\theta_{e}$ increases, the measured vertical size increases quickly due to the high aspect ratio of the electron beam and an angle of $17 \mathrm{~m}$ rad with an aspect ratio of 100:1 for example would double the measured electron beam size. $\sigma_{e y^{\prime}}=\sigma_{e y}$ when the angle of analysis matches the angle of the electron beam with respect to the laserwire. The reduced- $\chi^{2}, \sigma_{e y^{\prime}}$ and $\sigma_{e x^{\prime}}$ from fitting the data from Fig. 24 rotated by $\pm 12 \mathrm{mrad}$ are shown in Fig. 25 .

This shows a clear minimum with the smallest vertical size $\sigma_{e y}=1.702 \pm 0.023 \mu \mathrm{m}$ at an angle of $1.0 \mathrm{mrad}$. However, $\sigma_{e x^{\prime}}$ and $\sigma_{e y^{\prime}}$ show a different maximum and minimum, respectively, and are also asymmetric about the minimum, which is not to be expected from the simple roll of the electron beam. This is shown more explicitly in Fig. 26, where the model of Eq. (12) is shown with $\sigma_{e y^{\prime}}$. The fit of this model indicates a minimum vertical size of $\sigma_{e y^{\prime}}=1.727 \pm 0.007 \mu \mathrm{m}$ at an angle of $0.00 \pm 0.02 \mathrm{mrad}$, but this model clearly does not accurately describe $\sigma_{e y^{\prime}}\left(\theta_{e}\right)$.

Despite the minimum $\sigma_{e y^{\prime}}$ and maximum $\sigma_{e x^{\prime}}$ occuring at $\sim 0 \mathrm{mrad}$, the minimum reduced $-\chi^{2}$ is found at a greater angle of $-4 \mathrm{mrad}$, indicating a better fit to the data at this angle. These features are indicative of a further systematic effect not encapsulated by the model used. This is most likely due to the astigmatic propagation of the laser beam. Although the $M^{2}$ used accurately describes the laser propagation in terms of the diameter of the laser beam, 


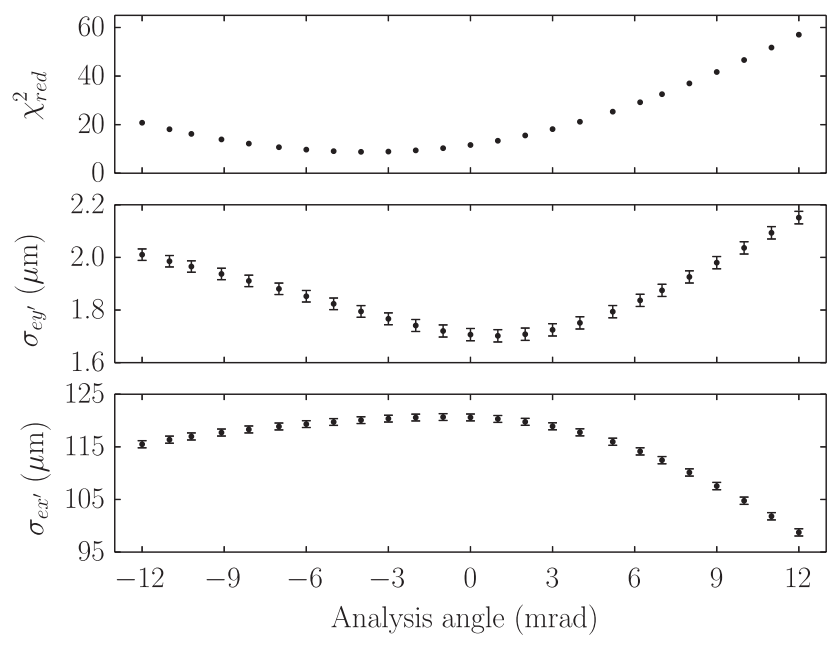

FIG. 25. Comparison of analyzed vertical and horizontal sizes from rotation of combined multiple laserwire scans as well as the reduced $-\chi^{2}$ for the fit in each case.

the transverse photon distribution is non-Gaussian and can be different on either side of the diffraction limited focus. This could be overcome with a laser with an improved transverse profile.

\section{Measured and predicted size}

During the laserwire operation period, the mOTR system was being upgraded and a comparative emittance measurement was not possible. The measured electron beam sizes with the laserwire agree very well with predicted sizes from the MAD8 model using the measured emittance by the laserwire and the measured dispersion.

The quadrupole scan made to measure the emittance using the laserwire was made at a different date than that of the smallest laserwire scan; however, they agree well. The predicted size using Eq. (1) is $1.129 \pm 0.021 \mu \mathrm{m}$ and the

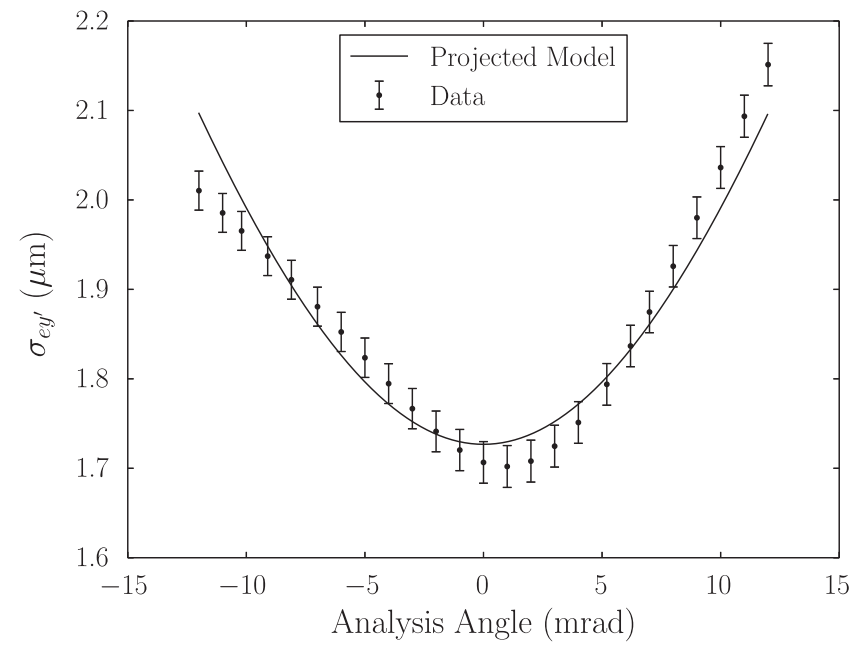

FIG. 26. Deconvolved vertical electron beam size as a function of analysis angle for combined multiple laserwire scans. The fit to a model of a projected bivariate Gaussian is also shown. measured size was $1.07_{-0.06}^{+0.06}$ (stat) \pm 0.05 (sys) $\mu \mathrm{m}$. The level of agreement shown is very good given that the optical functions at the extraction point in the damping ring were not measured during laserwire operations. Furthermore, given the $3.12 \mathrm{~Hz}$ repetition rate of the ATF2, further studies during one 8-hour operational shift are difficult.

\section{OUTLOOK}

The laserwire system described in this paper has demonstrated a high resolution, effectively noninvasive method of measuring the transverse profile of a high charge density, low emittance electron beam such as that at a future linear electron-positron collider. However, it is clear that several improvements could be made to reduce the measurement uncertainty.

\section{A. Scanning methods}

While the scanning methodology employed that consisted of a single vertical and horizontal scan was sufficient, it may not be optimal. From Fig. 21, it can be seen that a horizontal scan performed with a displacement of $\sim 15 \mu \mathrm{m}$ vertically would provide a scan with two maxima that may help to further constrain the fit to the model and reduce the uncertainty in the fitted parameters. Similarly, a coupled $x-y$ scan may provide further constraint. The nonlinear step size used in the scans was highly effective and can be adjusted to the approximate size of the electron beam.

In the laserwire described in this paper, only one laser beam is used, whereas in an ideal laserwire diagnostic station there would be two laser beam lines orthogonal to each other; one for the vertical and one for the horizontal beam size measurement [9]. In a similar case of an electron beam with a high aspect ratio, the major axis of the elliptical beam could be measured using a simple deconvolution requiring no knowledge of the minor axis size. This measurement of the major axis could then be used to deconvolve the laserwire scans of the smaller minor axis that may exhibit Rayleigh range effects as described in this paper. However, this would require knowledge of the precise horizontal offset of the laser focus with respect to the electron beam center and so a further horizontal scan would be required. As with the laserwire in this paper, it is possible to use a single laser beam to measure both profiles, but only where the horizontal beam size is significantly greater than the Rayleigh range of the laser beam. To this end, although one laser beam path may suffice, two would guarantee laserwire measurements over a larger range of electron beam sizes and aspect ratios.

\section{B. Vacuum chamber}

From the experiments conducted at the ATF2, it is possible to start the design process for a final ILC laserwire scanner. Our experimental vacuum chamber 
was engineered with flexibility and mechanical stability as the primary concerns, while a production ILC system will have to be optimized for cost and ease of operation. The authors would propose a smaller vacuum chamber, on a light weight mover system. Only a single station of the ILC emittance measurement system would require an OTR system as the relative timing could be set from a single measurement for other nearby stations. The small laserwire vacuum chamber would be surrounded by a small optical system to move the laser beam onto the final focus lens and safely deal with the outgoing laser beam. Hopefully, in the future the laser energy can be delivered to each station via a flexible fiber optic cable, without power loss and preserving the spatial and temporal quality of the beam.

\section{Laser source}

It is clear from the laser characterization that while the laser source used provides the necessary high peak power over a duration similar to the electron bunch length, the spatial quality could be improved. The laser source was astigmatic and had a non-Gaussian profile. In particular, the non-Gaussian nature makes the $M^{2}$ model an approximation of the size of the laser beam and not the true intensity distribution. A laser with a better spatial profile would adhere much more closely to the $M^{2}$ model and reduce the systematic uncertainty of the measured electron beam sizes [23].

Fiber lasers are a promising technology that could provide high spatial quality pulses at a much higher repetition rate with high overall efficiency. Recent work has focused on achieving the necessary high peak powers from optical fiber lasers for such applications as a laserwire [24]. Fiber lasers are not able to provide the gigawatt peak powers used for this laserwire system, but in a well characterized environment with a higher bunch charge electron beam such as a future linear collider, the peak power requirements would be considerably lower [6].

\section{Intratrain scanning}

For fast intratrain scanning the laser beam must be deflected in an angle, unlike the mechanical motion of the lens and chamber used at the ATF2 laserwire system. The shift in position of the focused laser spot is calculated using the deflection angle $\theta$ and the appropriate transfer function of the optical path $f(\theta)$. There are two main ways to angularly scan the laser beam; first, a piezo stack driven mirror [9] or an electro-optic scanner [25]. Alternatively, the possibility of dithering the position of the electron beam could be considered as the ranges vertically are in the $10 \mathrm{~s}$ of micrometer ranges, although the beam rigidity could make this impractical and would rely on precise pulse to pulse beam position information.

\section{E. Laser normalization}

For a more complete system, the beam charge and laser pulse energy need to be measured with uncertainties significantly less than the Compton or background signal variation. This is possible with high resolution direct current transformers for the particle beam, but the laser energy measurement systems would need more careful and systematic verification of performance before use with a laserwire system.

\section{F. BPMs for laserwires}

The number and location of BPMs in the vicinity of laserwire stations will be important in a multiple laserwire emittance measurement system. BPMs close to the laserwire interaction points are useful for quick spatial and temporal alignment after initial collisions have been found. To enable spatial position jitter subtraction, high resolution CBPMs will be required at each laserwire station. At the ATF2, the spatial position jitter corresponded to $\sim 0.2 \sigma$, which therefore puts a stringent requirement of a resolution $\ll 0.2 \mu$ m to make a statistical difference. Such resolutions have been demonstrated, but at the expense of dynamic range. Further study is required to understand how well a full system of CBPMs can be used together with a laserwire.

\section{G. Signal and background simulation}

For application of the laserwire to a high energy future linear collider, there will be many differences in the required setup for laserwire operations. In the case of a much higher energy electron beam, the Compton-scattered photons will receive a much larger fraction of the incoming electron energy, and it therefore may be easier to measure the loss of the degraded energy electrons from the accelerator lattice than the Compton-scattered photons, or a combination of both. Simulations of the exact accelerator lattice will help determine this and also optimize the detector design.

Maximizing the signal-to-noise ratio of the Comptonscattering rate measurement is key to improving the precision of the laserwire measurement and therefore minimizing the error in the emittance measurement. Although the electron beam optics developed for laserwire operations reduced the background significantly, the background signal level was still significant and limits the precision of the measurement. The effect of pointing jitter on the Compton-scattered photons as well as background conditions should be simulated and efforts to this end have been started using Beam Delivery Simulation (BDSIM) [26].

\section{CONCLUSIONS}

A laserwire capable of measuring high aspect ratio electron beams using a visible wavelength laser source 
has been demonstrated. A minimum vertical electron beam size of $1.07_{-0.06}^{+0.06}$ (stat) \pm 0.05 (sys) $\mu \mathrm{m}$ was measured with a corresponding horizontal beam size of $119.0_{-2.4}^{+2.4}$ (stat) \pm 0.01 (sys) $\mu \mathrm{m}$. A single quadrupole scan was used to measure the vertically projected geometric emittance of $82.56 \pm 3.04 \mathrm{pm} \mathrm{rad}$.

Simultaneous fitting of the data from the horizontal and vertical laserwire scans using the overlap integral model was demonstrated in the presence of Rayleigh range effects and was shown to provide an accurate measurement of both the horizontal and the vertical electron beam sizes. This has demonstrated that Rayleigh range effects do not preclude the use of a laserwire to measure a high aspect ratio beam. Furthermore, an alignment method capable of finding collisions between the laser and electron beam in under 20 min was developed.

The laserwire was successfully operated with a low electron bunch population of $0.2 \times 10^{10} \mathrm{e}^{-}$and will easily scale to $2 \times 10^{10} \mathrm{e}^{-}$. We have identified a series of improvements and studies, which could improve this diagnostic, reduce systematic uncertainties, and improve ease of engineering for a future laserwire. Overall, a diagnostic for a future linear collider such as the ILC has been demonstrated, capable of measuring an electron beam size of $1 \mu \mathrm{m}$.

\section{ACKNOWLEDGMENTS}

We would like to thank the ATF2 collaborators and staff for their help in achieving the necessary stable operating conditions to demonstrate the laserwire performance. Additional thanks to the CBPM group, the OTR group, and the SLAC team for many helpful discussions and help in characterizing the machine. The research leading to these results has received funding from the Science and Technology Facilities Council via the John Adams Institute, Royal Holloway University of London, and the University of Oxford. We would like to acknowledge CERN for financial support of this research within the CLIC-UK collaboration: Contract No. KE1870/DG/CLIC as well as under the FP7 Research Infrastructures project Eu-CARD, Grant Agreement No. 227579.

[1] M. Aicheler, P. Burrows, M. Draper, T. Garvey, P. Lebrun, K. Peach, and N. Phinney, CLIC Conceptual Design Report, CERN-2012-007, 2012.

[2] N. Phinney, N. Toge, and N. Walker, arXiv:0712.2361.

[3] M. Ross, S. Anderson, J. Frisch, K. Jobe, D. McCormick et al., AIP Conf. Proc. 648, 237 (2003).

[4] H. Hayano, in Proceedings of the 20th International Linac Conference, LINAC-2000, Monterey, CA (SLAC, Menlo Park, CA, 2000), pp. 146-148.

[5] P. Tenenbaum and T. Shintake, Annu. Rev. Nucl. Part. Sci. 49, 125 (1999).
[6] I. Agapov, G. Blair, and M. Woodley, Phys. Rev. ST Accel. Beams 10, 112801 (2007).

[7] M. Ross, in Proceedings of the 2003 Particle Accelerator Conference, Portland, OR (IEEE, New York, 2003), pp. 503-507.

[8] R. Alley et al., Nucl. Instrum. Methods Phys. Res., Sect. A 379, 363 (1996).

[9] T. Aumeyr et al., in Proceedings of the International Particle Accelerator Conference, Kyoto, Japan (ICR, Kyoto, 2010).

[10] S. Boogert, G. Blair, G. Boorman, A. Bosco, L. Deacon, P. Karataev, A. Aryshev, M. Fukuda, N. Terunuma, J. Urakawa, L. Corner, N. Delerue, B. Foster, D. Howell, M. Newman, R. Senanayake, R. Walczak, and F. Ganaway, Phys. Rev. ST Accel. Beams 13, 122801 (2010).

[11] Y. Honda, K. Kubo, S. Anderson, S. Araki, K. Bane, A. Brachmann, J. Frisch et al., Phys. Rev. Lett. 92, 054802 (2004).

[12] ATF2 Group, ATF2 Proposal, KEK Report No. 2005-2, 2005.

[13] G. White et al., Phys. Rev. Lett. 112, 034802 (2014).

[14] G. White, S. Molloy, A. Seryi, D. Schulte, R. Tomas, S. Kuroda, P. Bambade, and Y. Renier, in Proceedings of the 11th European Particle Accelerator Conference, EPAC 2008, Genoa, Italy (EPS-AG, Genoa, Italy, 2008), C0806233, TUPPO16.

[15] Y. Kim et al., Phys. Rev. ST Accel. Beams 15, 042801 (2012).

[16] T. Johnston, Appl. Opt. 37, 4840 (1998).

[17] P. Karataev, A. Aryshev, S. Boogert, D. Howell, N. Terunuma, and J. Urakawa, Phys. Rev. Lett. 107, 174801 (2011).

[18] Experimental physics and industrial control system, http:// www.aps.anl.gov/epics/.

[19] J. Alabau-Gonzalvo, C. Blanch Gutierrez, A. Faus-Golfe, J. Garcia-Garrigos, J. Resta-Lopez et al., in Proceedings of the 2nd International Particle Accelerator Conference, San Sebastián, Spain (EPS-AG, Spain, 2011), pp. 879-881.

[20] ISO 11146-2:2005 Lasers and laser-related equipmentTest methods for laser beam widths, divergence angles and beam propagation ratios-Part 2: General astigmatic beams, 2005, http://www.iso.org/iso/catalogue_detail .htm?csnumber $=33626$.

[21] F. James and M. Roos, Comput. Phys. Commun. 10, 343 (1975).

[22] S. Y. Lee, Accelerator Physics (World Scientific, Singapore, 2004), 2nd ed.

[23] C. J. Saraceno, F. Emaury, C. Schriber, M. Hoffmann, M. Golling, T. Südmeyer, and U. Keller, Opt. Lett. 39, 9 (2014).

[24] L. J. Nevay, R. Walczak, and L. Corner, Phys. Rev. ST Accel. Beams, 17, 072801 (2014).

[25] A. Bosco, S. Boogert, G. Boorman, and G. Blair, Appl. Phys. Lett. 94, 211104 (2009).

[26] L. Deacon and G. Blair, Laser Wire Simulation in the ILC Beam Delivery System, EUROTEV Report No. 2008-018. 